\title{
Reaction Forces on a Fixed Ladder in Static Equilibrium: Analysis and Definitive Experimental Test of the Ladder Problem
}

\author{
M. P. Silverman \\ Department of Physics, Trinity College, Hartford, USA \\ Email: mark.silverman@trincoll.edu
}

How to cite this paper: Silverman, M.P. (2018) Reaction Forces on a Fixed Ladder in Static Equilibrium: Analysis and Definitive Experimental Test of the Ladder Problem. World Journal of Mechanics, 8, 311-342. https://doi.org/10.4236/wjm.2018.89024

Received: August 2, 2018

Accepted: August 27, 2018

Published: August 30, 2018

Copyright $\odot 2018$ by author and Scientific Research Publishing Inc. This work is licensed under the Creative Commons Attribution International License (CC BY 4.0).

http://creativecommons.org/licenses/by/4.0/

Open Access

\begin{abstract}
The development of a theoretical model to predict the four equilibrium forces of reaction on a simple ladder of non-adjustable length leaning against a wall has long remained an unresolved matter. The difficulty is that the problem is statically indeterminate and therefore requires complementary information to obtain a unique solution. This paper reports 1) a comprehensive theoretical analysis of the three fundamental models based on treating the ladder as a single Euler-Bernoulli beam, and 2) a detailed experimental investigation of the forces of reaction as a function of applied load and location of load. In contrast to previous untested proposals that the solution to the ladder problem lay in the axial constraint on compression or the transverse constraint on flexure, the experimental outcome of the present work showed unambiguously that 1) the ladder could be modeled the best by a pinned support at the base (on the ground) and a roller support at the top (at the wall), and 2) the only complementary relation needed to resolve the static indeterminacy is the force of friction at the wall. Measurements were also made on the impact loading of a ladder by rapid ascent and descent of a climber. The results obtained were consistent with a simple dynamical model of the ladder as a linear elastic medium subject to a pulse perturbation. The solution to the ladder problem herein presented provides a basis for theoretical extension to other types of ladders. Of particular importance, given that accidents involving ladders in the workplace comprise a significant fraction of all industrial accidents, the theoretical relations reported here can help determine whether a collapsed structure, against which a ladder was applied, met regulatory safety limits or not.
\end{abstract}

\section{Keywords}

Reaction Forces on a Ladder, Reaction Forces on a Beam, Impulse Load on a 
Ladder, Ladder in Static Equilibrium, Statically Indeterminate Forces

\section{Introduction: The Ladder Problem}

Although determination of the reaction forces on a beam is a standard part of the mechanics of continuous media, the exact conditions under which an actual physical structure comprised of beams is stressed are not always apparent. Models based on different assumptions can predict hugely different reaction forces, and careful experimentation is required to determine which model may most accurately characterize a structure. One of the most important mechanical structures for which reaction forces need to be determined is that of a ladder. Although ladders have been in use since antiquity, to date no definitive model of the reaction forces on a ladder has been experimentally tested and confirmed. This paper reports what the author believes to be the most comprehensive theoretical analysis and definitive experimental test of the reaction forces on a fixed ladder.

\subsection{The Fixed Ladder as an Euler-Bernoulli Beam}

A fixed ladder is defined by the American Ladder Institute as a non-self-supporting ladder of fixed (i.e. non-adjustable) length [1]. It comprises two identical rails of uniform density connected by short rungs. As a suitable first approximation to modeling such a ladder, the rungs are ordinarily disregarded and the two rails are merged into a single uniform beam, such as shown in panel A of Figure 1. The single-beam ladder of length $L$ is in static equilibrium supported obliquely by the flat horizontal ground and a flat vertical wall. The weight $W$ of the ladder is regarded as a uniformly distributed load of linear density

$$
w=W / L
$$

that acts vertically downward at the center of mass of any isolated segment of the beam. A climber is modeled as a point load $P$ acting vertically downward at a distance $\beta L \quad(1 \geq \beta \geq 0)$ along the beam from the contact point at the ground, which is taken to be the origin.

As shown in panel A of the figure, there are four reaction forces: 1) normal force at the ground $\left.\left(R_{1}\right), 2\right)$ parallel force at the ground $\left(R_{2}\right), 3$ ) normal force at the wall $\left(R_{3}\right)$, and 4$)$ parallel force at the wall $\left(R_{4}\right)$. The prediction of these four forces constitutes what is called the ladder problem. Before discussing the theoretical solutions and experimental test of the ladder problem, it is worthwhile to consider why the problem is a significant one. This significance is both conceptual and practical.

First, conceptually, the solution to any model serving as an archetype for a class of real-world problems within some branch of physics is significant by virtue of its fundamentality. The single-beam ladder is an archetype within the mechanics of continuous media. Moreover, understanding the forces on a fixed 


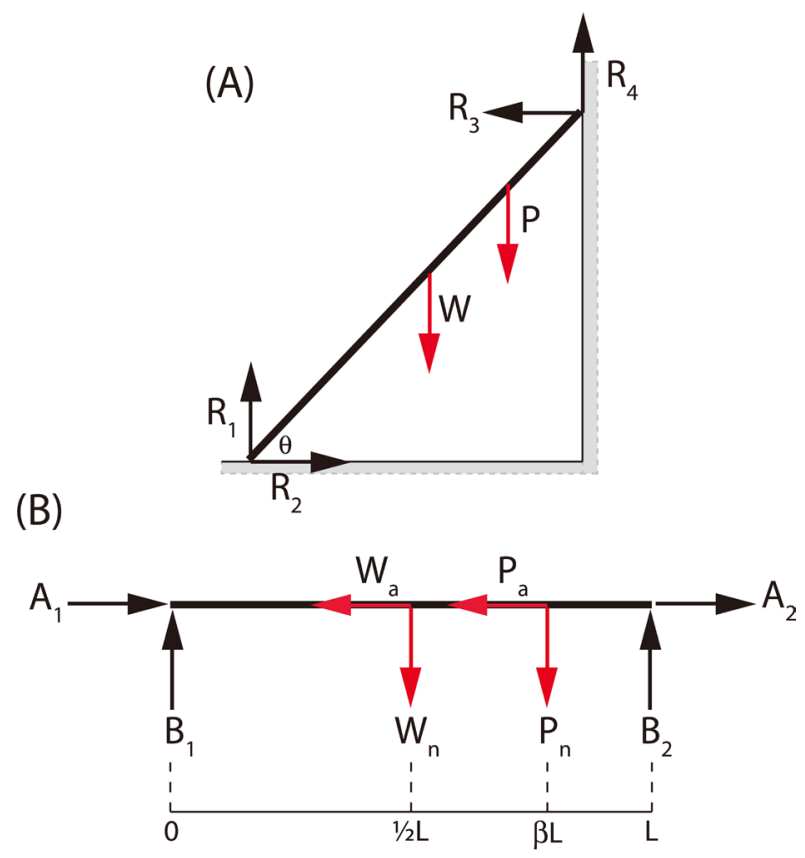

(C)

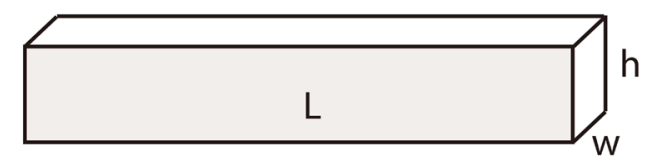

Figure 1. (A) Schematic diagram of the reaction forces $\left(R_{1}, R_{2}, R_{3}, R_{4}\right)$ on a single-beam ladder by the ground and wall. $W$ is the beam weight acting at the center of mass $L / 2 ; P$ is a point load applied at the point $\beta L$; (B) Decomposition of forces to show axial $\left(A_{1}, A_{2}\right)$ and normal $\left(B_{1}, B_{2}\right)$ components relative to the long axis of the beam. Subscript 1 denotes the contact point at the ground; subscript 2 denotes the contact point at the wall.

ladder would provide useful modeling guidelines for predicting the reaction forces on more complex ladder types, such as extension ladders, step ladders, trestle ladders, and others. Second, from a practical standpoint, the ability to predict the forces on ladders has direct impact on matters relating to safety in the use of ladders and to legal issues that arise when use of a ladder in the workplace results in injury or death. For example, with a valid fixed ladder model, the analyst can determine the acceptable range of inclination angles to avoid slippage, or determine whether the net force exerted by a ladder on a wall or railing that collapsed was within or exceeded regulatory limits [2].

One might think that prediction of the reaction forces on a structure as simple as a single-beam ladder is a relatively straightforward matter. Indeed, the problem is trivially solvable if the parallel reaction at the wall is ignored, which is the case in countless physics textbooks (see, for example, [3]) and expository internet articles. The fixed ladder leaning against a frictionless wall has been an iconic example of static equilibrium in elementary mechanics books for at least a century. This problem is statically determinate; i.e. the three reaction forces 
$\left(R_{1}, R_{2}, R_{3}\right)$ are uniquely solvable from the three equations of static equilibrium in which the net horizontal force, net vertical force, and rotational moment about any point at rest all vanish. The resulting solution, however, is of academic interest only and does not describe correctly the interactions of real ladders with real surfaces.

When account is taken of the parallel reaction at the wall, the problem becomes statically indeterminate, and therefore more complicated, since there are now 4 unknowns and 3 equations. One proposed solution assumed that the ladder undergoes an axial deformation which is constrained by the supports [4]. This model cannot be correct as it stands because the transverse (bending) deformation-as calculated in Section 2-is approximately two orders of magnitude greater than the axial deformation and should not have been neglected. Another proposal [5] took account of both axial compression and transverse flexion, and concluded that the problem could not be solved by static analysis because it was not possible to determine which deformation dominated. This conclusion is not substantiated by the findings of the present paper. Neither of these proposals provided experimental measurements to test their validity.

Experimentally, there have been many investigations, of which some representative examples are [6] [7] [8], to elucidate the conditions under which ladders can be used safely. In general, these investigations focused on matters relating to friction, angle of inclination, climbing speed, weight of climber, and other empirically accessible variables, but did not attempt to provide a model that would succinctly incorporate these data in a mathematical theory.

This paper reports the theoretical solution of three basic models by which to analyze the reaction forces of a fixed ladder represented as a single Euler-Bernoulli (E-B) beam. The characteristics of an E-B beam pertinent to the present study are as follows [9]:

1) the length of the beam is much greater than the linear dimension of the cross-section;

2) the long axis of the beam lies within the neutral surface;

3) the cross section of the beam remains plane and perpendicular to the long axis during deflection;

4) deformation of the cross section within its own plane is neglected;

5) the beam is a linear elastic material subject to Hooke's law; i.e. normal stress within a cross section varies linearly with perpendicular distance from the neutral axis.

The neutral surface of a beam is the interface that separates the fibers under compression from the fibers under tension when the beam is deflected transversely. The neutral axis within any cross section is the line of intersection of the neutral surface with the cross section.

\subsection{The Analytical Models}

The three basic ladder models analyzed in this paper are distinguished by the nature of the supports at the points of contact with the surfaces of the ground 
and wall. In the mechanics of beams, the three common types of supports are 1) roller, 2) pin, and 3) fixed [10]. The names refer to the constraints on the motion of the affected segment of the beam, and not necessarily to the actual presence of a roller, pin, or clamping mechanism. Thus, if one end of a beam is supported by a roller, it can displace laterally, but not vertically. If the end of the beam is pinned, it cannot displace either laterally or vertically, but it can rotate about the pin. And, finally, if the end is fixed, it cannot displace or rotate at all. [There is a fourth kind of support usually designated as simple, which is idealized as a frictionless surface. This would apply to the support of the single-beam ladder at a wall as treated in the elementary physics books.]

If a right-handed Cartesian coordinate system $(x, y, z)$ is chosen such that an initially horizontal beam lies along the $x$ axis, and a load is applied that can deflect the beam vertically downward along the negative $y$ axis, then the boundary and continuity conditions at the three kinds of supports can be summarized as follows

$$
\begin{array}{ll}
\text { Roller: } & \Delta y=0, \mathrm{~d} y / \mathrm{d} x \neq 0, M_{z}=0 \\
\text { Pin: } & \Delta y=0, \mathrm{~d} y / \mathrm{d} x \neq 0, M_{z}=0 \\
\text { Fixed: } & \Delta y=0, \mathrm{~d} y / \mathrm{d} x=0, M_{z} \neq 0
\end{array}
$$

where the symbol $\Delta$ signifies displacement, and $M_{z}$ is an internal bending moment (torque) about the $z$ axis. The salient features of relations (2) are that a) the displacement and slope of the deflected beam are zero at a fixed support but the support creates an internal bending moment, whereas b) at roller and pin supports there is no internal moment, and the slope of the bending curve there need not be zero.

The models analyzed in this paper are based on the supports described by Equation (2).

- In Model 1, the ground support of the single-beam ladder is fixed and the wall support is equivalent to a pin. Under a vertical load due to the distributed weight of the ladder and the point weight of a hypothetical climber, the ladder is subject to both axial and transverse stresses, but is constrained from compression, elongation, or rotation at the supports. This is a case of static indeterminateness in two variables: one axial and one transverse. Although friction is presumably the source of the constraints, no specific assumptions are made in regard to the mathematical form of the frictional force.

- In Model 2, the ground support of the single-beam ladder is fixed, and the wall support is equivalent to a roller. The axial component of the load can compress the ladder, and thereby remove the static indeterminateness along the length. The reaction at the wall is assumed to be governed by the phenomenological relation for friction taken to be proportional to the normal force at the wall [11]. There remains a static indeterminateness in one of the transverse reactions because of the unknown internal moment at the ground support.

- In Model 3, the ground support of the single-beam ladder is equivalent to a 
pin and the wall support is equivalent to a roller. The ladder is free to compress under an axial force and flex about the ground support, thereby relieving all static constraints. The reaction at the wall is assumed governed by friction as in Model 2. This is a case of static indeterminateness in one variable in which the equations of static equilibrium, complemented by the frictional force law, suffice to determine a unique solution.

The three models are schematically illustrated in Figure 2.

\subsection{Outline of Paper}

In Section 2 the reaction forces on a ladder characterized as a single E-B beam are calculated for each model and examined as a function of load, location of load along the ladder, and angle of inclination of the ladder with respect to the ground. In the case of Models 1 and 3, the full bending curve is calculated within the Euler-Bernoulli approximation (neglect of the square of the slope $\mathrm{d} y / \mathrm{d} x$ ), and the magnitudes of transverse and axial deformations are compared. (The bending curve of Models 2 and 1 are the same).

In Section 3 are reported experiments using horizontal and vertical force platforms to measure the reactions on a fixed metal ladder inclined against a wall. It is noted here briefly that the model that was found to account for the observed results was Model 3.

In Section 4 the impact loading on a ladder by a climber is examined theoretically and experimentally.

In Section 5 are reported measurements of the reactions on a wood beam inclined obliquely against a wall. The intent of the experiment was to ascertain whether the same theoretical model that best applied to a ladder modeled as a beam applied as well to an actual single beam.

In Section 6 conclusions drawn from this research are summarized.
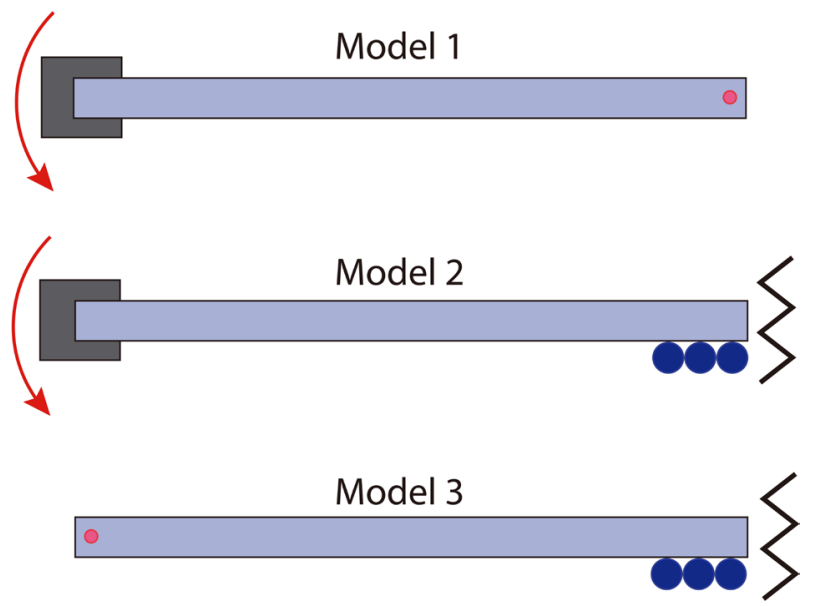

Figure 2. Schematic diagram symbolizing the supports upon which each model is based: gray clamp = fixed support; red solid circle $=$ pin support; 3 blue circles $=$ roller. The fixed support is accompanied by an internal moment (red arc). The zigzag line symbolizes friction. The light blue bar represents the single-beam ladder as portrayed horizontally in panel B of Figure 1. 


\section{Analysis of a Single-Beam Fixed Ladder}

Panel A of Figure 1 shows the disposition of the reaction forces on the ladder as it appears inclined against a vertical wall at an angle $\theta$ to the ground. Because the material out of which an actual ladder is constructed-primarily aluminum, fiberglass, or wood-has a relatively high elastic modulus ( $E_{\mathrm{Al}} \sim 69 \mathrm{GPa}$, $\left.E_{\mathrm{FG}} \sim 72 \mathrm{GPa}, E_{\text {wood }} \sim 10-13 \mathrm{GPa}\right)$ [12] [13] [14], the deformation is ordinarily small, and one can analyze the axial and transverse deformations independently provided the axial force is not excessively large [15]. Thus, by taking appropriate components, one obtains the axial and transverse reaction forces shown in panel B of Figure 1, which are related to the forces normal and parallel to the ground and wall as follows

$$
\begin{aligned}
& A_{1}=R_{1} \sin \theta+R_{2} \cos \theta \\
& B_{1}=R_{1} \cos \theta-R_{2} \sin \theta \\
& A_{2}=-R_{3} \cos \theta+R_{4} \sin \theta \\
& B_{2}=R_{3} \sin \theta+R_{4} \cos \theta
\end{aligned}
$$

with inverse relations

$$
\begin{aligned}
& R_{1}=B_{1} \cos \theta+A_{1} \sin \theta \\
& R_{2}=-B_{1} \sin \theta+A_{2} \cos \theta \\
& R_{3}=-B_{2} \sin \theta-A_{2} \cos \theta \\
& R_{4}=B_{2} \cos \theta+A_{2} \sin \theta
\end{aligned}
$$

The equations of static equilibrium then take either of the forms

$$
\text { (I) } \begin{aligned}
& R_{1}+R_{4}=W+P \\
& R_{2}-R_{3}=0
\end{aligned} \quad \text { (II) } \begin{aligned}
& B_{1}+B_{2}=W_{n}+P_{n} \\
& A_{1}+A_{2}=W_{a}+P_{a}
\end{aligned}
$$

in which the normal and axial components of the ladder weight and a point load representative of a climber are respectively

$$
\begin{array}{ll}
W_{n}=W \cos \theta & W_{a}=W \sin \theta \\
P_{n}=P \cos \theta & P_{a}=P \sin \theta .
\end{array}
$$

The sets of equations in (5) do not contain an equation for the vanishing of a rotational moment about a designated point at rest. In the absence of information regarding the supports of the ladder at the ground, it is not possible to write a torque equation based on static equilibrium alone. This is a consequence of the fact that the system may contain an initially unknown internal moment created by the ground support in order to maintain static equilibrium.

Since there are four unknown reactions in Equation (5) and fewer than four equations of static equilibrium, the set of equations is statically indeterminate and must be supplemented by what are termed complementary conditions. Under the circumstances stated above, these conditions can be applied to the axial and normal directions independently. The complementary conditions take different forms depending on the specific model, as discussed in the following subsections. 


\subsection{Model 1: Fixed Ground and Pinned Wall Supports}

With respect to the schematic diagram in panel B of Figure 1, Model 1 entails a fixed support at the left end $x=0$ (with initially unknown internal moment $M_{z}(0)$ ) and a pin support at the right end $x=L$. The total strain energy $U$ of the beam

$$
U=U_{a}+U_{b}
$$

is the sum of the axial strain energy

$$
U_{a}=\frac{1}{2 E A} \int_{0}^{L} F_{a}(x)^{2} \mathrm{~d} x
$$

due to the compression or tension by force $F_{a}(x)$ and the energy of flexure (bending)

$$
U_{b}=\frac{1}{2 E I_{z}} \int_{0}^{L} M_{z}(x)^{2} \mathrm{~d} x
$$

due to the moment $M_{z}(x)$ about the $z$ axis (see Ref. [15], pp 188-196). The material parameters in Equations (8) and (9) are the beam cross section area $A$, beam area moment of inertia $I_{z}$ about the transverse $z$ axis, and the elastic modulus (Young's modulus) E. For a beam of width $w$ and height $h$ as portrayed in panel $\mathrm{C}$ of Figure 1, the cross section and area moment of inertia take the forms (see [15], pp 448-449)

$$
\begin{gathered}
A=w h \\
I_{z}=\frac{1}{12} w h^{3} .
\end{gathered}
$$

Because the overall load comprises both distributed and point contributions, the force $F_{a}(x)$ and moment $M_{z}(x)$ are piecewise continuous functions. These functions can be represented economically by use of the Heaviside unit step function [16] defined here as

$$
H\left(x-x_{0}\right)= \begin{cases}0 & x<x_{0} \\ 1 & x \geq x_{0}\end{cases}
$$

Mathematically, the Heaviside function is usually defined by pure inequalities in both partitions of the real axis and left undefined at the point where the argument is zero. In the mechanics of continuous media, however, the point load occurs at the partition between segments and therefore it is more useful to define the Heaviside function as unity there. One can then express the axial force and bending moment in the following ways

$$
\begin{gathered}
F_{a}(x)=A_{2}-\frac{W_{a}}{L}(L-x)-P_{a} H(\beta L-x) \\
M_{z}(x)=B_{2}(L-x)-\frac{W_{n}}{2 L}(L-x)^{2}-P_{n}(\beta L-x) H(\beta L-x) .
\end{gathered}
$$

From the complementary energy theorem (Castigliano's theorem) of continuum mechanics (see [15] pp 201-217) for systems where the force-deformation 
relation is linear, the deflection of a point $i$ on a beam in the direction of a load $F_{i}$ applied at that point is given by

$$
\delta_{i}=\frac{\partial U}{\partial F_{i}} .
$$

Since a physical condition of Model 1 is that the right endpoint of the beam in panel B of Figure 1 suffers no deflection, it follows that

$$
\delta_{L}^{(a)}=\frac{\partial U}{\partial A_{2}}=0
$$

and

$$
\delta_{L}^{(b)}=\frac{\partial U}{\partial B_{2}}=0
$$

where superscript $a$ signifies axial and superscript $b$ signifies bending. Equations (16) and (17) are the complementary relations required to supplement the set of relations (5) and resolve the static indeterminacy of Model 1.

Substitution of relations (13) and (14) respectively into strain energies (8) and (9), taking derivatives (16) and (17), and setting the resulting integrals to zero lead to expressions for the axial reaction $A_{2}$ and transverse reaction $B_{2}$. The reactions $A_{1}$ and $B_{1}$ are then obtained from Equation (5) of static equilibrium. The resulting set of four axial and transverse reactions are found to be

$$
\begin{aligned}
& A_{1}=\frac{1}{2} W \sin \theta+(1-\beta) P \sin \theta \\
& A_{2}=\frac{1}{2} W \sin \theta+\beta P \sin \theta \\
& B_{1}=\frac{5}{8} W \cos \theta+\left(1-\frac{3}{2} \beta^{2}+\frac{1}{2} \beta^{3}\right) P \cos \theta \\
& B_{2}=\frac{3}{8} W \cos \theta+\left(\frac{3}{2} \beta^{2}-\frac{1}{2} \beta^{3}\right) P \cos \theta
\end{aligned}
$$

from which follow by transformation (4) the reactions normal and parallel with respect to the ground and wall

$$
\begin{aligned}
& R_{1}=\frac{1}{2} W\left(1+\frac{1}{4} \cos ^{2} \theta\right)+P\left[1-\left(\frac{3}{2} \beta^{2}-\frac{1}{2} \beta^{3}\right) \cos ^{2} \theta-\beta \sin ^{2} \theta\right] \\
& R_{2}=R_{3}=\left[\frac{1}{16} W+\left(\frac{1}{2} \beta-\frac{3}{4} \beta^{2}+\frac{1}{4} \beta^{4}\right) P\right] \sin 2 \theta \\
& R_{4}=\frac{1}{2} W\left(1-\frac{1}{4} \cos ^{2} \theta\right)+P\left[\left(\frac{3}{2} \beta^{2}-\frac{1}{2} \beta^{3}\right) \cos ^{2} \theta+\beta \sin ^{2} \theta\right] .
\end{aligned}
$$

The directions of $R_{2}$ and $R_{3}$ are shown in Figure 1, and no algebraic sign denoting direction is needed since these two forces are not superposed in any calculation in this paper.

Figure 3 shows the variation in normal force at the ground (panel A) and at the wall (panel $\mathrm{B}$ ) as a function of the fractional location $(1 \geq \beta \geq 0)$ of the applied load along the beam for different load magnitudes ranging from 0 to 100 
pounds (lbs). The ladder weight is taken to be $25 \mathrm{lbs}(111.2 \mathrm{~N})$, in accordance with the use of English units in ladder specifications given in the US, but calculations are performed and recorded in metric units (newtons $\mathrm{N}$ ). The angle of inclination was taken to be $60^{\circ}$. Plots of the parallel force at the ground $\left(R_{2}\right)$ and at the wall $\left(R_{4}\right)$ are not shown since by relations (18) the former are the same as the plots in panel $\mathrm{B}$, and the latter correspond to reversing the horizontal scale (abscissa) in panel A.

A complementary perspective is given in Figure 4, which shows the variation in normal force at the ground (panel A) and at the wall (panel B) as a function of the angle of inclination for different fractional locations of the applied load along the beam. According to Model 1, the location of a hypothetical climber (of
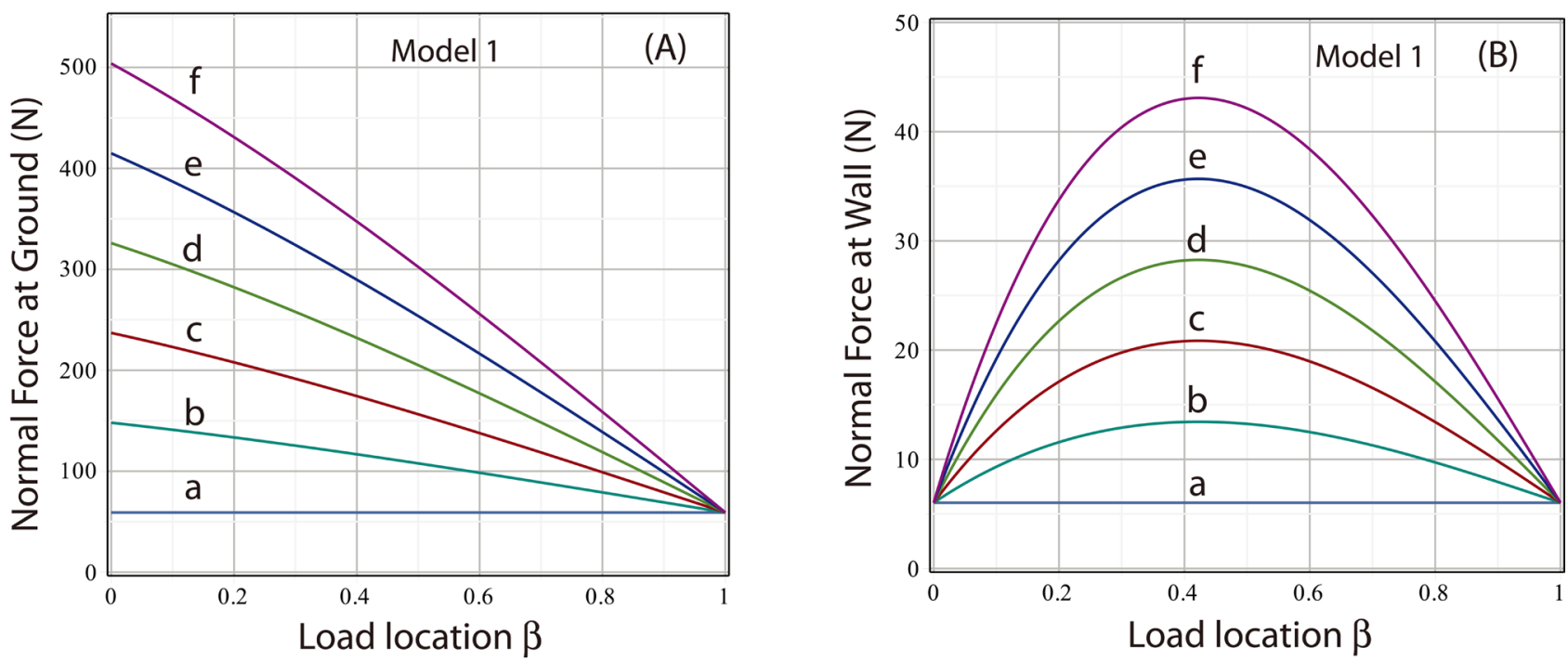

Figure 3. Model 1 variation in normal force at (A) ground and (B) wall as a function of load location for loads (in lbs): (a) 0, (b) 20 , (c) 40, (d) 60, (e) 80, (f) 100. The ordinate unit is in newtons. Ladder weight is 25 lbs; angle of inclination is $60^{\circ}$.
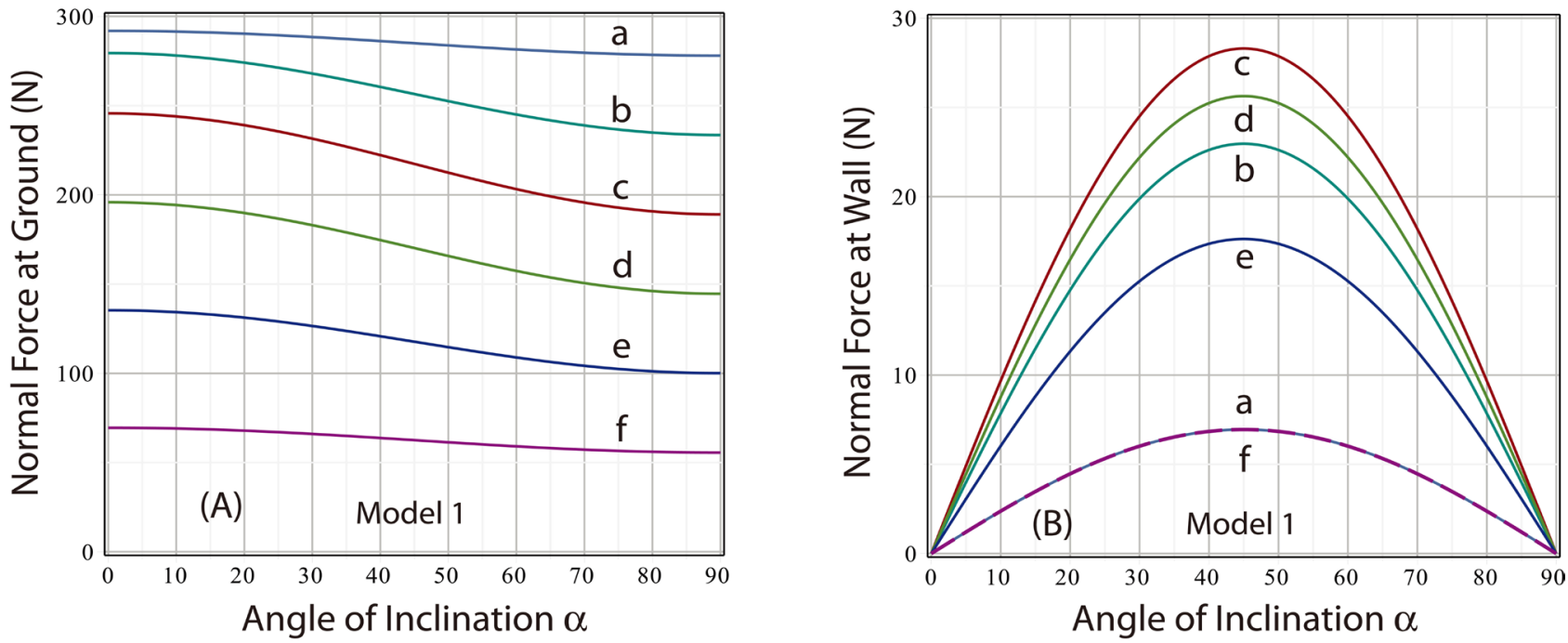

Figure 4. Model 1 variation in normal force at (A) ground and (B) wall as a function of inclination angle for load locations $\beta$ : (a) 0.0 , (b) 0.2 , (c) 0.4 , (d) 0.6, (e)).8, (f) 1.0. Ladder weight is $25 \mathrm{lbs}$; point load is $50 \mathrm{lbs}$. 
fixed weight) influences the normal reaction at the wall strongly and the normal reaction at the ground relatively weakly as the inclination of the ladder is changed.

Applying Equation (15) to the total strain energy $U$, one can determine the deformation at any point in the elastic curve of the single-beam ladder and thereby compare the magnitudes of the axial compression $\delta^{(a)}$ and transverse (bending) deflection $\delta^{(b)}$. Taking $\beta=\frac{1}{2}$ for illustration yields the deformations

$$
\begin{gathered}
\delta_{\frac{1}{2} L}^{(a)}=\left.\frac{\partial U}{\partial P_{a}}\right|_{\beta=\frac{1}{2}}=\frac{L}{2 A E}(W+P) \sin \theta \\
\delta_{\frac{1}{2} L}^{(b)}=\left.\frac{\partial U}{\partial P_{n}}\right|_{\beta=\frac{1}{2}}=\frac{L^{3}}{768 E I_{z}}(4 W+7 P) \cos \theta .
\end{gathered}
$$

Given typical parameters of beam length $L=2.5 \mathrm{~m}$, width $w=2 \mathrm{~cm}$, height $h=5 \mathrm{~cm}$, inclination angle $\theta=45^{\circ}$, ladder weight $W=25 \mathrm{lbs}(111.2$ $\mathrm{N})$, climber weight $P=150 \mathrm{lbs}(667.2 \mathrm{~N})$, and elastic modulus of aluminum $E=69 \mathrm{GPa}$, Equations (20) and (21) lead to deformations $\delta_{\frac{1}{2} L}^{(a)} \approx 10 \mu \mathrm{m}$, $\delta_{\frac{1}{2} L}^{(b)} \approx 5.1 \mathrm{~mm}$. The deformation due to bending is approximately 500 times the deformation due to axial compression. Therefore, any model of a ladder subject to ordinary loads that takes account of axial deformation cannot neglect deformation due to flexure.

As a final consideration, it is useful to derive the deflection curve of Model 1 as a check on the calculated deformations and to ascertain that the reactions of a single-beam ladder derived from Model 1 correctly fulfill the complementary conditions. The exact flexure formula for an E-B beam relates the bending moment to the radius of curvature and takes the form of a nonlinear second-order differential equation (see [10] pp. 576-583)

$$
\frac{\mathrm{d}^{2} y / \mathrm{d} x^{2}}{\left[1+(\mathrm{d} y / \mathrm{d} x)^{2}\right]^{3 / 2}}=\frac{1}{E I_{z}} M_{z}(x)
$$

whose solution is called the elastica [17]. Although an exact solution would be required for a highly flexible beam such as a fishing rod [9], the deflection of the rail of a ladder is sufficiently low that one can neglect the square of the slope in the denominator of Equation (22), as is conventionally done in engineering practice. The equation then becomes linear and can be readily solved subject to the imposed boundary conditions

$$
\begin{aligned}
& y(0)=y(L)=0 \\
& y(x) \text { is continuous at } x=\beta L \\
& \mathrm{~d} y / \mathrm{d} x \text { is continuous at } x=\beta L
\end{aligned}
$$

to yield the piecewise continuous function 


$$
y(x)=y_{1}(x) H(\beta L-x)+y_{2}(x) H(x-\beta L)
$$

with

$$
\begin{aligned}
y_{1}(x)_{\text {Model } 1}= & \frac{1}{E I_{z}}\left[-L\left(\frac{1}{16} W_{n}+\left(\frac{1}{2} \beta-\frac{3}{4} \beta^{2}+\frac{1}{4} \beta^{3}\right) P_{n}\right) x^{2}\right. \\
& \left.+\left(\frac{5}{48} W_{n}+\frac{1}{6}\left(1-\frac{3}{2} \beta^{2}+\frac{1}{2} \beta^{3}\right) P_{n}\right) x^{3}-\frac{W_{n}}{24 L} x^{4}\right] \\
y_{2}(x)_{\text {Model } 1}= & \frac{1}{E I_{z}}\left[\frac{1}{6} \beta^{3} L^{3} P_{n}-\frac{1}{2}\left(\beta^{2} L^{2} P_{n}\right) x+L\left(-\frac{1}{16} W_{n}+\left(\frac{3}{4} \beta^{2}-\frac{1}{4} \beta^{3}\right) P_{n}\right) x^{2}\right. \\
& \left.+\left(\frac{5}{48} W_{n}-\frac{1}{6}\left(\frac{3}{2} \beta^{2}-\frac{1}{2} \beta^{3}\right) P_{n}\right) x^{3}-\frac{W_{n}}{24 L} x^{4}\right] .
\end{aligned}
$$

Figure 5 shows plots of the deflection curve $y(x)$ for different locations of a $150 \mathrm{lb}$ load on a ladder $2.5 \mathrm{~m}$ in length and $25 \mathrm{lbs}$ in weight inclined at an angle of $45^{\circ}$ against a vertical wall. The cross section of the single beam is $2 \mathrm{~cm} \times 5 \mathrm{~cm}$ and the modulus of elasticity is $E=69 \mathrm{GPa}$. As seen in the figure, the deflection curve is the same for a load directly at the base $x=0$ or at the top of the ladder $x=L$. The deflection $\delta_{\frac{1}{2} L}^{(b)} \sim 5.1 \mathrm{~mm}$ occurs at the mid-position $\beta=\frac{1}{2}$, which is the result predicted by Equation (21) obtained by Castigliano's theorem. The deflection curve is not symmetric for any location of the load, even at mid-position, because the slope is required to be 0 at the ground support (left end) but not at the wall support (right end).

Calculation of deflections (20), (21) and the elastica curve (24) involved

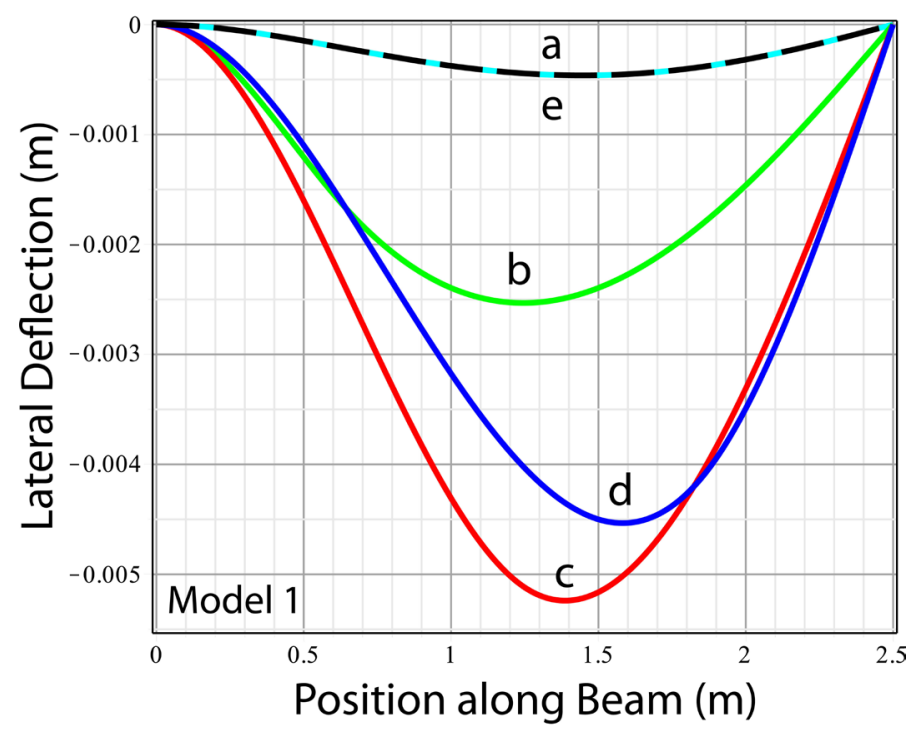

Figure 5. Deflection curves of the Model 1 single-beam ladder of length $2.5 \mathrm{~m}$ and weight $25 \mathrm{lbs}$ for a point load of $150 \mathrm{lbs}$ at fractional lengths $\beta$ along the beam of (a) 0 [ground point], (b) $1 / 4$, (c) $1 / 2$, (d) $3 / 4$, (e) 1 [wall point] (note that plots (a) (solid line) and (e) (dashed line) overlap). The inclination angle of the ladder was taken to be $45^{\circ}$. Material parameters are $E=69 \mathrm{GPa}, A=2 \times 5 \mathrm{~cm}^{2}, I_{z}=\frac{1}{12}(2 \mathrm{~cm})(5 \mathrm{~cm})^{3}$ 
integration of expressions of the form $x^{n} H\left(x-x_{0}\right)$ for non-negative integer $n$. The integration of Heaviside functions is summarized in the Appendix.

\subsection{Model 2: Fixed Ground and Roller Wall Supports}

With respect to the schematic diagram in panel B of Figure 1, Model 2 entails a fixed support at the left end and a roller support at the right end. Resolution of the static indeterminateness of the bending reactions is accomplished in exactly the same way as for Model 1 , and therefore forces $B_{1}$ and $B_{2}$ are given as shown in Equations (18). To determine the other forces, one makes use of the transformation (3) for $B_{2}$ and the phenomenological relation for friction

$$
R_{4}=\mu R_{3}
$$

with coefficient of friction $\mu$ to obtain

$$
B_{2}=R_{3} \sin \theta+R_{4} \cos \theta=R_{3}(\sin \theta+\mu \cos \theta),
$$

from which follows

$$
R_{3}=R_{2}=\frac{R_{4}}{\mu}=\frac{\frac{3}{8} W+\left(\frac{3}{2} \beta^{2}-\frac{1}{2} \beta^{3}\right) P}{\tan \theta+\mu}
$$

and therefore from Equation (5)

$$
R_{1}=W+P-R_{4}=W+P-\mu R_{3}=W+P-\mu\left[\frac{\frac{3}{8} W+\left(\frac{3}{2} \beta^{2}-\frac{1}{2} \beta^{3}\right) P}{\tan \theta+\mu}\right] .
$$

The axial forces $A_{1}$ and $A_{2}$ are given by the transformation (3)

$$
\begin{aligned}
& A_{1}=(W+P) \sin \theta+\left(\frac{3}{8} W+\left(\frac{3}{2} \beta^{2}-\frac{1}{2} \beta^{3}\right) P \cos \theta\right)\left[\frac{1-\mu \tan \theta}{\tan \theta+\mu}\right] \\
& A_{2}=-\left(\frac{3}{8} W+\left(\frac{3}{2} \beta^{2}-\frac{1}{2} \beta^{3}\right) P \cos \theta\right)\left[\frac{1-\mu \tan \theta}{\tan \theta+\mu}\right]
\end{aligned}
$$

rather than by a complementary condition constraining axial compression.

Figure 6 shows the variation in normal force at the ground (panel A) and at the wall (panel B) as a function of load location for different loads under the same conditions as for Figure 3. The coefficient of friction was taken to be 0.4. Correspondingly, Figure 7 shows the variation in these two reactions as a function of angle of inclination with the ground under the same conditions as for Figure 4. Models 1 and 2 are seen to predict reactions that depend very differently on load location.

Since the reaction forces normal to the beam $\left(B_{1}, B_{2}\right)$ are the same as for Model 1, the bending deflection curve, which does not depend on the axial reactions, is also the same.

\subsection{Model 3: Pinned Ground and Roller Wall Supports}

With respect to the schematic diagram in panel B of Figure 1, Model 3 entails a 

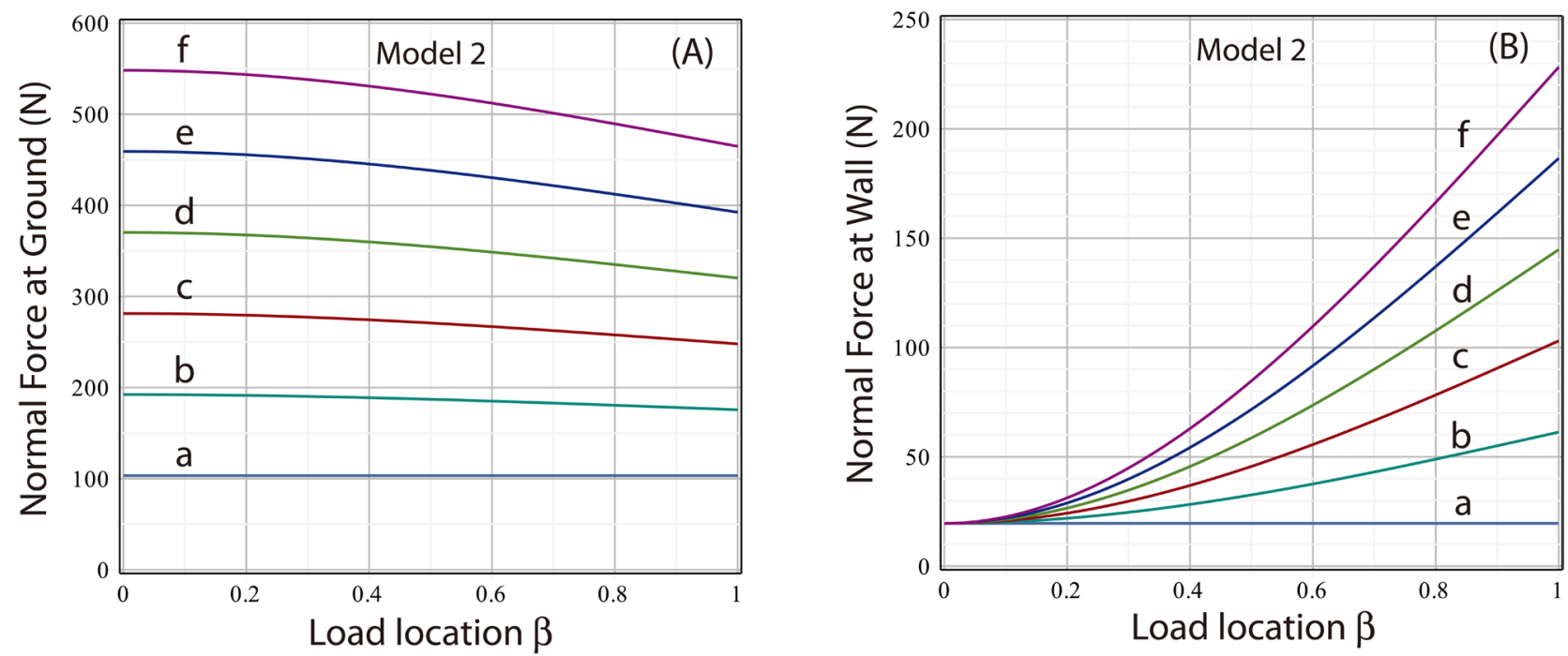

Figure 6. Model 2 variation in normal force at (A) ground and (B) wall as a function of load location for loads (in lbs): (a) 0, (b) 20, (c) 40, (d) 60, (e) 80, (f) 100. The weight and angle are the same as in Figure 3; the coefficient of friction is 0.4.
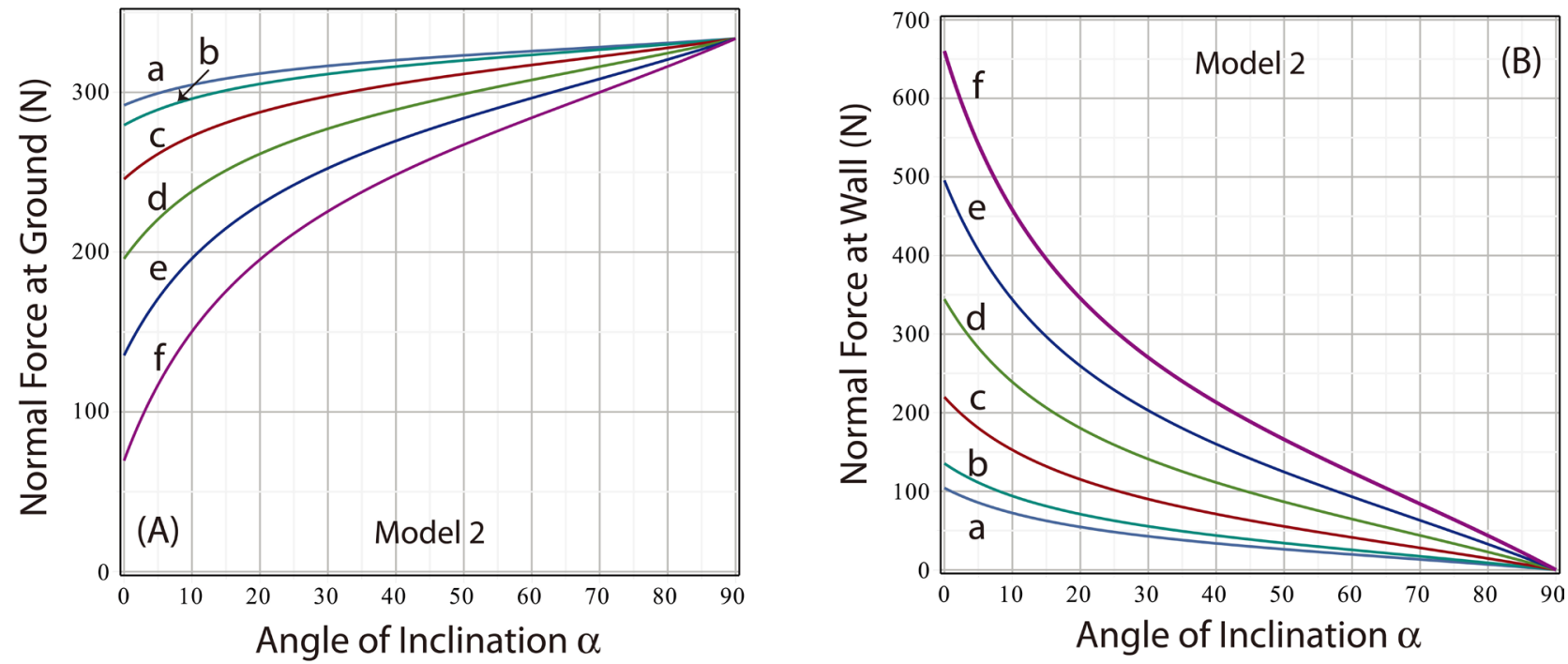

Figure 7. Model 2 variation in normal force at (A) ground and (B) wall as a function of inclination angle for load locations $\beta$ : (a) 0.0 , (b) 0.2 , (c) 0.4, (d) 0.6, (e)).8, (f) 1.0. The weight and load are the same as in Figure 4; the coefficient of friction is 0.4 .

pinned support at the left end and a roller support at the right end. Because there is no unknown internal moment at pinned and roller supports, the set of equations (5) can be supplemented by an equation for the vanishing of the external moment $M_{z}$ about any fixed point. For example, if the pivot is taken to be the point of contact with the ground, then the vanishing of $M_{z}(0)$ can be expressed by either of the equations

$$
R_{3} \sin \theta+R_{4} \cos \theta=\frac{1}{2} W \cos \theta+\beta P \cos \theta
$$

or

$$
B_{2}=\frac{1}{2} W_{n}+\beta P_{n} .
$$


Together with the complementary relation (26) for force of friction and the other equations of static equilibrium, one obtains

$$
\begin{gathered}
R_{3}=R_{2}=\frac{R_{4}}{\mu}=\frac{\frac{1}{2} W+\beta P}{\tan \theta+\mu} \\
B_{1}=\frac{1}{2} W_{n}+(1-\beta) P_{n}
\end{gathered}
$$

and

$$
R_{1}=W+P-\mu R_{3}=\frac{W\left(\tan \theta+\frac{1}{2} \mu\right)+P(\tan \theta+(1-\beta) \mu)}{\tan \theta+\mu} .
$$

From the set of transformations (3) follow the axial reactions

$$
\begin{aligned}
& A_{1}=(W+P) \sin \theta+\left(\frac{3}{8} W+\left(\frac{3}{2} \beta^{2}-\frac{1}{2} \beta^{3}\right) P \cos \theta\right)\left[\frac{1-\mu \tan \theta}{\tan \theta+\mu}\right] \\
& A_{2}=-\left(\frac{3}{8} W+\left(\frac{3}{2} \beta^{2}-\frac{1}{2} \beta^{3}\right) P \cos \theta\right)\left[\frac{1-\mu \tan \theta}{\tan \theta+\mu}\right]
\end{aligned}
$$

Figure 8 and Figure 9 respectively show the variation in normal force at the ground (panel A) and at the wall (panel B) as a function of load location along the ladder and as a function of inclination angle with the ground. The plots are similar to, but not numerically identical with, the plots of Figure 6 and Figure 7 .

The bending curve (elastica) of Model 3 takes a different shape than that of Models 1 and 2 because of the difference in the nature of the support at the ground. Solving the Euler-Bernoulli approximation to the flexure equation (22)

$$
\mathrm{d}^{2} y / \mathrm{d} x^{2}=M_{z}(x) / E I_{z}
$$

with moment (see panel B of Figure 1)

$$
M_{z}(x)=B_{1} x-\frac{W_{n}}{2 L} x^{2}-P_{n}(x-\beta L) H(x-\beta L)
$$

and boundary conditions (23) leads to the piecewise continuous function (24) with components

$$
\begin{aligned}
y_{1}(x)_{\text {Model } 3}= & \frac{1}{E I_{z}}\left[-L^{2}\left(\frac{1}{24} W_{n}+\left(\frac{1}{3} \beta-\frac{1}{2} \beta^{2}+\frac{1}{6} \beta^{3}\right) P_{n}\right) x\right. \\
& \left.+\left(\frac{1}{12} W_{n}+\frac{1}{6}(1-\beta) P_{n}\right) x^{3}-\frac{W_{n}}{24 L} x^{4}\right] \\
y_{2}(x)_{\text {Model } 3}= & \frac{1}{E I_{z}}\left[\frac{\beta^{3} L^{3}}{6} P_{n}-L^{2}\left(\frac{1}{24} W_{n}+\left(\frac{1}{3} \beta+\frac{1}{6} \beta^{3}\right) P_{n}\right) x+\left(\frac{1}{2} \beta L P_{n}\right) x^{2}\right. \\
& \left.+\left(\frac{1}{12} W_{n}-\frac{1}{6} \beta P_{n}\right) x^{3}-\frac{W_{n}}{24 L} x^{4}\right]
\end{aligned}
$$

Figure 10 shows plots of the deflection curve $y(x)$ for different locations of a $150 \mathrm{lb}$ load on a ladder $2.5 \mathrm{~m}$ in length and $25 \mathrm{lbs}$ in weight inclined at an angle of $45^{\circ}$ against a vertical wall. As seen in the figure, the deflection curve is again 
the same for a load directly at the base $x=0$ or at the top of the ladder $x=L$. In contrast to the deflection curves of Models 1 and 2, the deflection curve of Model 3 is symmetric for a load applied at the middle of the beam; the slopes are nonzero and equal at both the ground and wall contact points. For other locations of the load, the deflection curve is no longer symmetric, but at no location are the slopes zero at the end points.

The maximum deflection $\delta_{\frac{1}{2} L}^{(b)} \sim 11.8 \mathrm{~mm}$ occurs at the mid-position $\beta=1 / 2$. This is the same result one would get from applying Castigliano's theorem, as implemented in Section 2.1, which leads to the deformation
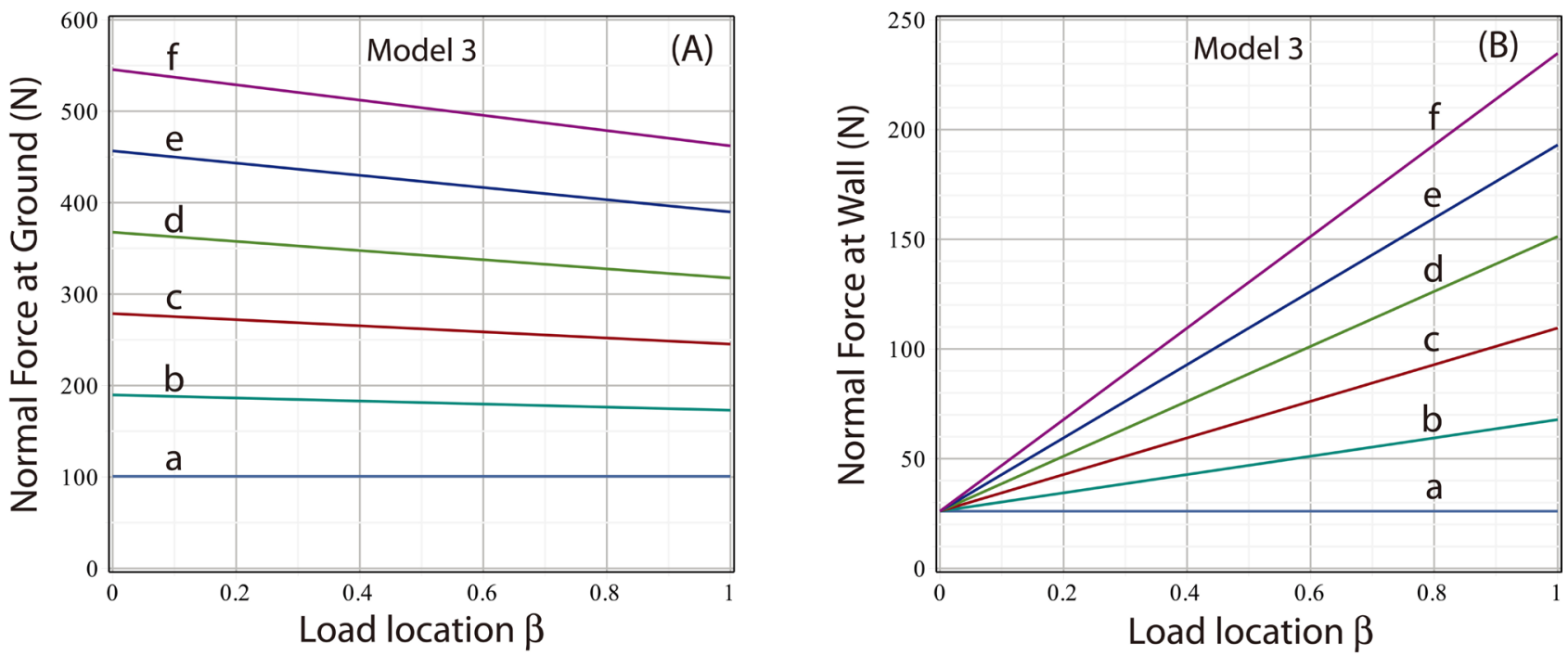

Figure 8. Model 3 variation in normal force at (A) ground and (B) wall as a function of load location for loads (in lbs): (a) 0, (b) 20, (c) 40, (d) 60, (e) 80, (f) 100. The weight and angle are the same as in Figure 3; the coefficient of friction is 0.4 .
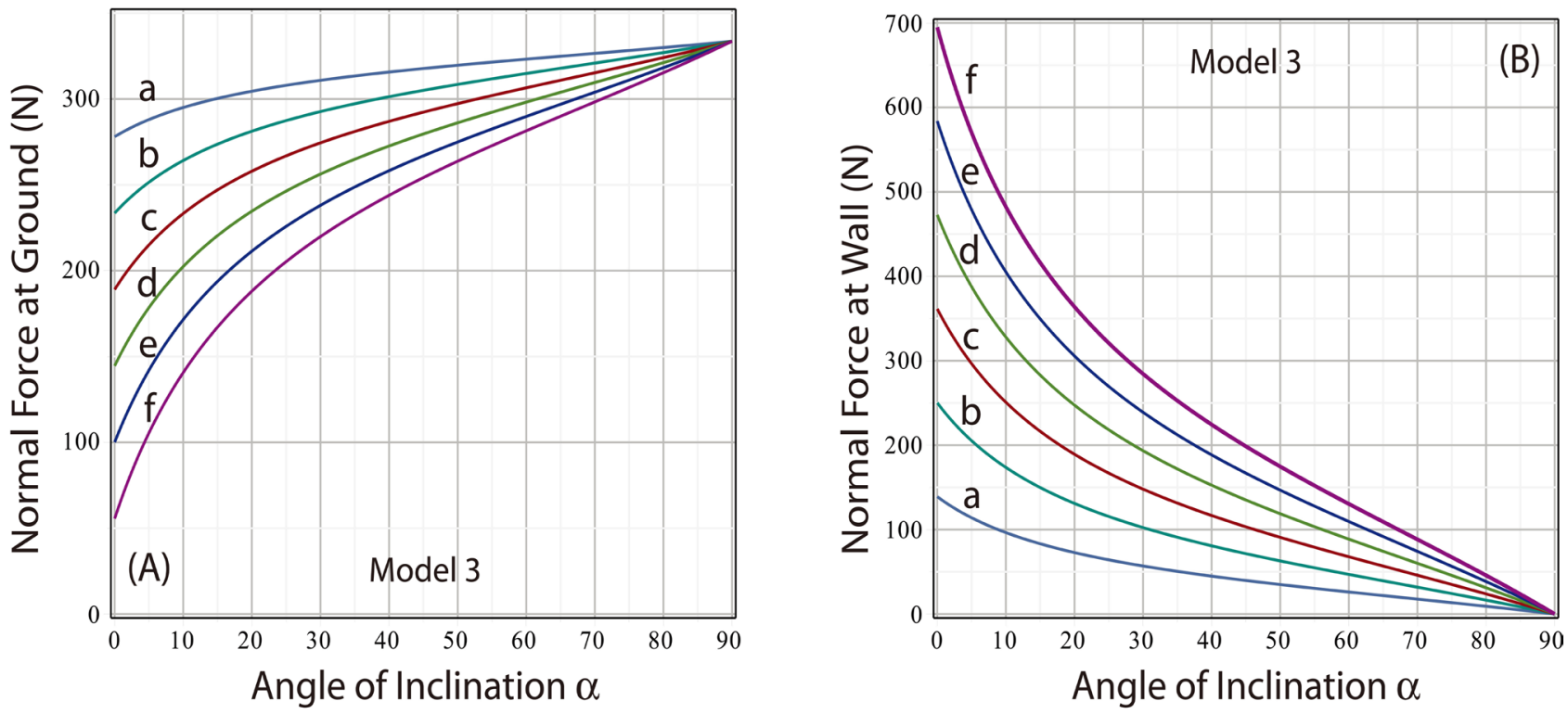

Figure 9. Model 3 variation in normal force at (A) ground and (B) wall as a function of inclination angle for load locations: (a) 0.0, (b) 0.2 , (c) 0.4 , (d) 0.6, (e)).8, (f) 1.0. The weight and load are the same as in Figure 4; the coefficient of friction is 0.4. 


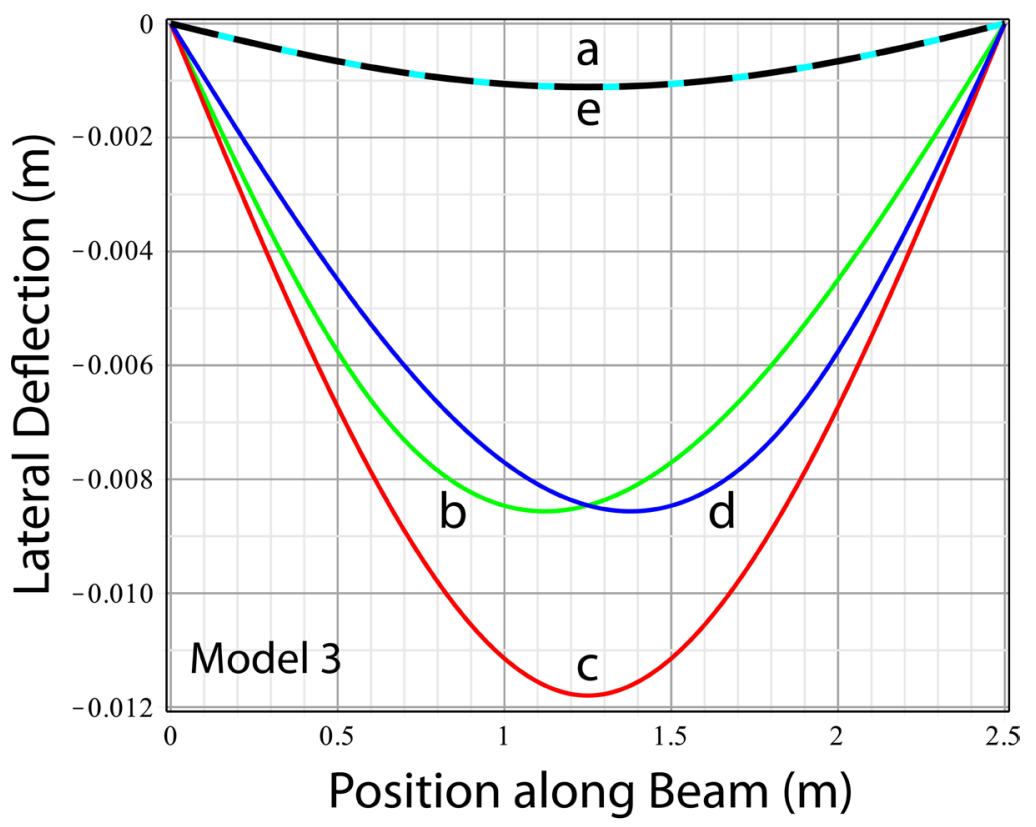

Figure 10. Deflection curves of the Model 3 single-beam ladder under the same conditions as for Figure 5 for fractional lengths $\beta$ along the beam of (a) 0 [ground point], (b) $1 / 4$, (c) $1 / 2$, (d) $3 / 4$, (e) 1 [wall point] (note that plots (a) (solid line) and (e) (dashed line) overlap). The coefficient of friction is 0.4 .

$$
\delta_{\frac{1}{2} L}^{(b)}=\frac{L^{3}}{384 E I_{z}}(5 W+8 P) \cos \theta .
$$

The deflection produced by the axial forces of Equation (36) is given by

$$
\delta_{\beta L}^{(a)}=\frac{L}{A E}\left(A_{2}-\left(\frac{1}{2} W+\beta P\right) \sin \theta\right)\left(\frac{1-\mu \tan \theta}{\tan \theta+\mu}\right) .
$$

For the load at mid-position and the other parameters of Figure 10, Equation (41) leads to an axial compression $13.3 \mu \mathrm{m}$. Thus, as was the case with Model 1, the bending deflection is again seen to be at least two orders of magnitude greater than the corresponding deformation due to axial compression.

\section{Measured Reaction Forces on a Fixed Ladder}

To determine which, if any, of the three basic models describes most closely the reaction forces on a fixed ladder, an experiment was performed to measure forces $R_{1}, R_{2}$, and $R_{3}$. The experiment was performed as follows. A ladder of length $244 \mathrm{~cm}$ and weight $18.9 \mathrm{lbs}(84.07 \mathrm{~N})$ was placed against a vertical wall at an angle of $68^{\circ}$ to the ground. A two-axis force platform (Pasco Model PS-2142) was situated horizontally on the ground beneath each of the two rails of the ladder. The top of the ladder rested on a third force platform attached vertically to the wall. Each force platform, used in conjunction with a computer and the appropriate interface, was designed to measure the applied force normal to its surface (up to a maximum of $4400 \mathrm{~N}$ ) and along an axis parallel to its surface (up to $1100 \mathrm{~N})$. 
The ladder comprised seven rungs located at fractional lengths along the rails of

$$
\begin{aligned}
\beta & =\left(\frac{32}{244}, \frac{62}{244}, \frac{92}{244}, \frac{122}{244}, \frac{152}{244}, \frac{182}{244}, \frac{212}{244}\right) \\
& \approx(0.131,0.254,0.377,0.500,0.623,0.746,0.869)
\end{aligned}
$$

of which the first six rungs were used in the experiment. External loads were applied to the rungs in the form of lead bricks, each brick weighing $25 \mathrm{lbs}$ (111.21 $\mathrm{N})$. Measurements of the normal and parallel forces were made for discrete loads of $1,2,3$, or 4 bricks placed on each rung in progressive order from rung 1 to rung 6 . The sum of the forces normal to the horizontal surface of force platforms 1 and 2 (supporting the rails) constituted reaction $R_{1}$. The sum of the forces parallel to the surface of platforms 1 and 2 constituted reaction $R_{2}$. The force normal to the vertical surface of force platform 3 (against which the top of the ladder rested) constituted reaction $R_{3}$. Although the initial intention was to measure reaction $R_{4}$ as well, this measurement could not be made because the force platforms did not measure shear forces accurately when the platform was oriented vertically ${ }^{1}$. The omitted reaction, however, was not needed to establish the conclusions drawn from this experiment.

Table 1 records the measurements of the 3 reactions $\left(R_{1}, R_{2}, R_{3}\right)$ at each of 6 rungs for each of 4 loads $(25,50,75,100 \mathrm{lbs})$. The experimental forces are respectively compared with the corresponding theoretical predictions of the three models in Figures 11-13.

In order to apply the theoretical relations of Models 2 and 3, it is necessary to know the coefficient of friction $\mu$ of the ladder with the wall-or, more accurately, with the surface of the force platform attached to the wall. This coefficient cannot be calculated theoretically, but must be obtained empirically from the data. The method employed here was trial and inspection; i.e. to assume a value for $\mu$, calculate and plot the resulting reactions, and adjust as necessary the value of $\mu$ until a satisfactory match with data was achieved, provided that the model itself is a valid representation of the underlying mechanical processes. The entire procedure can be executed in a matter of seconds with a fast computer and available mathematical software such as Maple or Mathcad. It is to be emphasized that the totality of the 72 measurements ( 3 reactions $\times 4$ loads $\times 6$ load locations) tightly constrained the value of $\mu$. For Model 2 or 3 to be valid it is necessary that a single value of $\mu$ suffice to account for all the data. If different statistically significant values of $\mu$ for the various reactions are needed to match the data, or if no value of $\mu$ matches the data, then Model 2 or 3 would not describe the static equilibrium of a ladder against a wall.

\footnotetext{
${ }^{1} \mathrm{~A}$ force normal to the surface of the platform is measured by means of 4 beams connecting the 4 footpads of the device to strain gauges. A force parallel to the surface (oriented along an indicator arrow) is measured by a single strain gauge connected to the center of the upper surface. Small deviations from center in placement of the top of the ladder resulted in erratic values of the parallel force.
} 
Table 1. Experimental Normal and Parallel Reactions on a Ladder.

\begin{tabular}{|c|c|c|c|c|c|}
\hline$\theta=68.0^{\circ}$ & LOAD (lbs) & 25 & 50 & 75 & 100 \\
\hline REACTIONS (N) & RUNG & & & & \\
\hline \multicolumn{6}{|l|}{ Ground Normal $R_{1}$} \\
\hline & 1 & 347.81 & 303.74 & 412.54 & 526.04 \\
\hline & 2 & 188.54 & 302.98 & 413.19 & 526.67 \\
\hline & 3 & 193.31 & 304.92 & 409.82 & 522.06 \\
\hline & 4 & 189.95 & 303.37 & 413.52 & 520.52 \\
\hline & 5 & 187.31 & 300.74 & 409.77 & 516.84 \\
\hline & 6 & 188.50 & 298.47 & 403.50 & 508.11 \\
\hline \multicolumn{6}{|l|}{ Ground Parallel $R_{2}$} \\
\hline & 1 & 20.19 & 23.87 & 31.56 & 37.00 \\
\hline & 2 & 22.92 & 35.01 & 47.29 & 58.86 \\
\hline & 3 & 32.47 & 48.67 & 63.63 & 80.60 \\
\hline & 4 & 37.15 & 60.90 & 86.58 & 106.12 \\
\hline & 5 & 37.50 & 67.05 & 95.88 & 121.87 \\
\hline & 6 & 47.62 & 81.22 & 113.40 & 145.17 \\
\hline \multicolumn{6}{|l|}{ Wall Normal $R_{3}$} \\
\hline & 1 & 10.01 & 14.09 & 17.70 & 21.40 \\
\hline & 2 & 16.45 & 28.49 & 36.74 & 46.61 \\
\hline & 3 & 24.14 & 39.44 & 53.67 & 69.85 \\
\hline & 4 & 28.82 & 51.80 & 74.75 & 95.94 \\
\hline & 5 & 32.76 & 60.15 & 87.46 & 112.59 \\
\hline & 6 & 40.07 & 73.52 & 104.20 & 138.55 \\
\hline
\end{tabular}

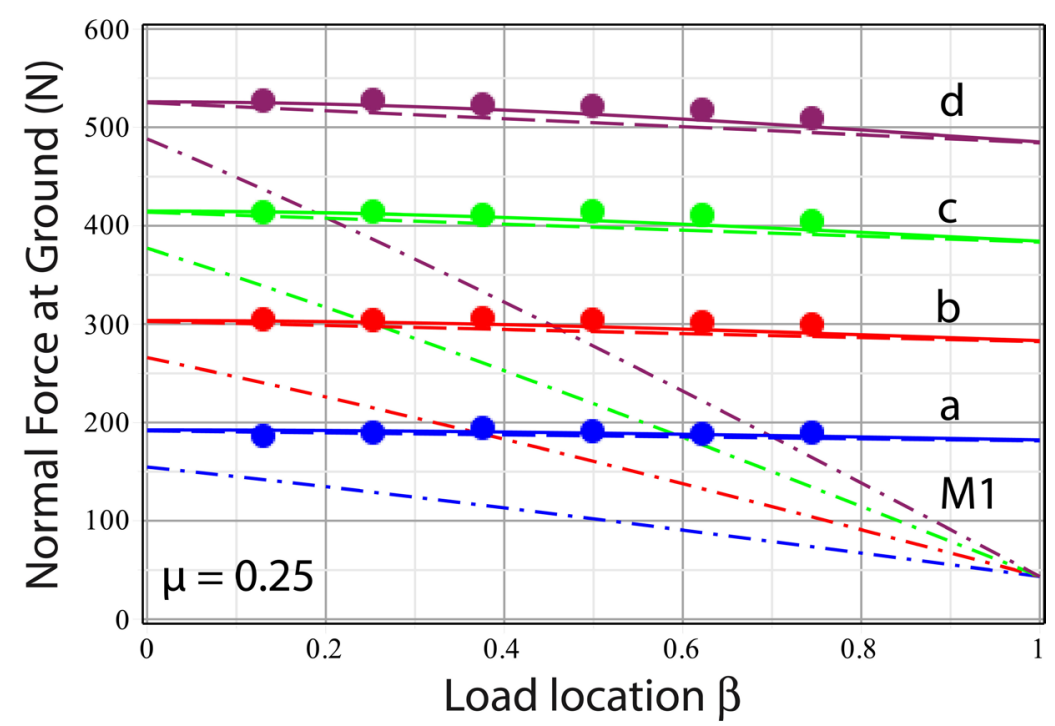

Figure 11. Experimental measurement (solid circles) of the normal reaction $R_{1}$ at the ground compared with predictions of Model 1 (dash-dot), Model 2 (solid), and Model 3 (dash) for loads (in lbs) of (a) 25 (blue), (b) 50 (red), (c) 75 (green), (d) 100 (burgundy). The set of Model 1 curves designated M1 do not match the data at all. 


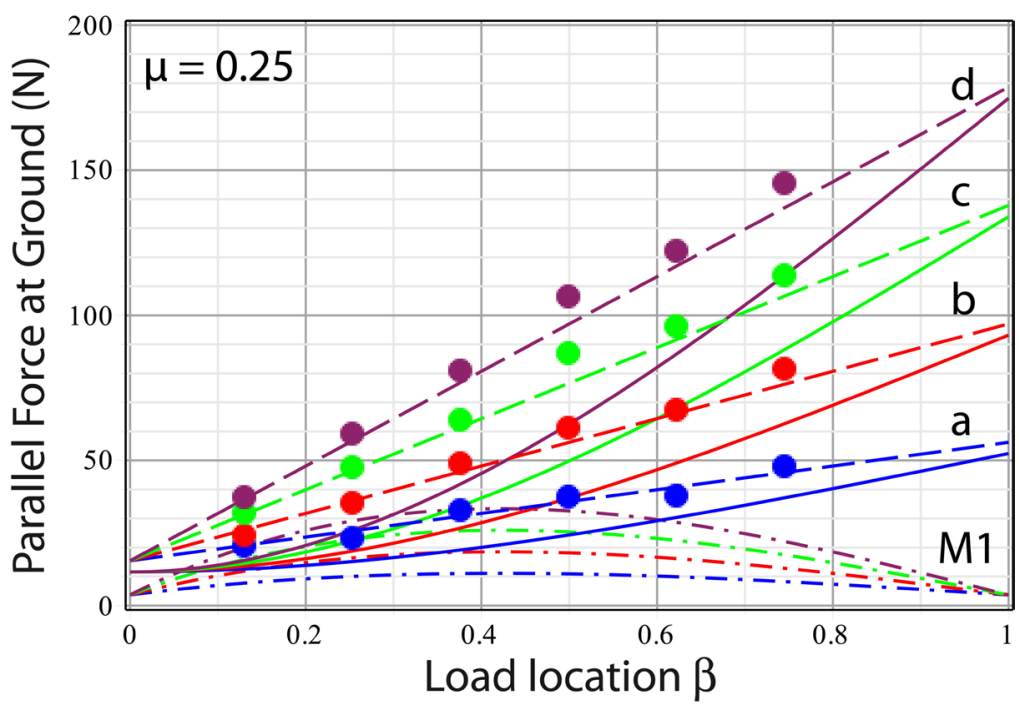

Figure 12. Experimental measurement of the parallel reaction $R_{2}$ at the ground compared with predictions of the three models. The symbolism in color and line style is the same as for Figure 11. Model 3 best matches the data.

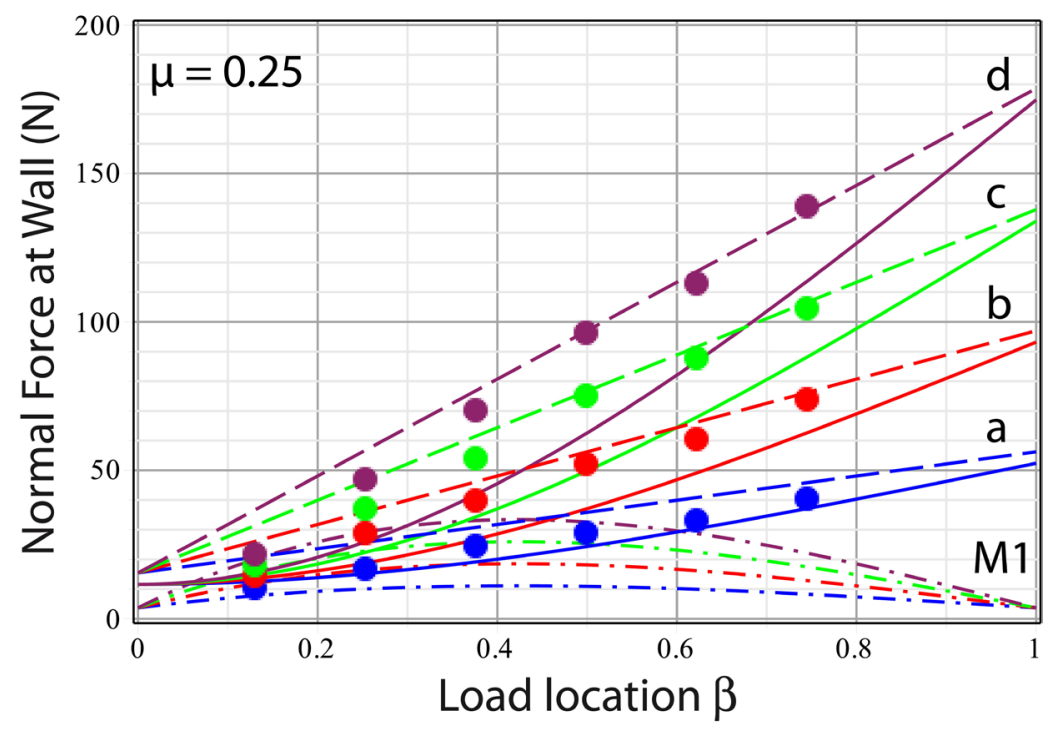

Figure 13. Experimental measurement of the normal reaction $R_{3}$ at the wall compared with predictions of the three models. The symbolism in color and line style is the same as for Figure 11. Model 3 best matches the data.

From examination of Figures 11-13 one can conclude the following:

- Model 1 does not account for any of the measured reactions.

- Models 2 and 3 account more or less equally well for the normal reaction $R_{1}$ at the ground support (Figure 11).

- Model 2 does not account for the measurements of the parallel reaction $R_{2}$ at the ground (Figure 12) or normal reaction $R_{3}$ at the wall (Figure 13).

- Model 3 accounts satisfactorily for all three reaction forces for $\mu \approx 0.25$ estimated from the data by trial and inspection.

To test the consistency of Model 3, the reaction parallel to the wall $R_{4}$ was 
obtained from the data by the two independent relations

$$
\begin{aligned}
& \text { (a) } R_{4}=\mu R_{3} \\
& \text { (b) } R_{1}+R_{4}=W+P
\end{aligned}
$$

where relation (a) is one of the defining conditions of the model, and relation (b) is required by Newton's 2nd law applied to static equilibrium. Panel A of Figure 14 shows plots of $R_{4}$ for different loads and load locations obtained from relation (a) of Equation (42). Of the three models, Model 3 is again seen to account most closely for the patterns of measured points. Panel B of Figure 14 plots relation (b) of Equation (42) with $R_{4}$ given by independent relation (a). As expected, the expression $R_{1}+R_{4}$ sums to a constant equal to $W+P$ for each of the four values of applied load $P$.

\section{Interpretation of Results}

From the foregoing comprehensive experimental test, one can conclude that, of the various mechanical conditions presumed to describe the static equilibrium of a ladder against a wall, those upon which Model 3 is based best represent the actual physical situation. It is useful to summarize explicitly the nature of these conditions.

- The elimination of Model 1 indicates that the length of the ladder is not constrained by axial forces at the ground or wall. Recall that it was this constraint that provided a complementary relation for resolving static indeterminateness in the axial direction. However, the reported experiment is consistent with no unrelieved axial strain. This means that the ladder, in response to its own weight and/or to the applied load, has relaxed to its (very slightly) compressed length in the course of reaching static equilibrium.
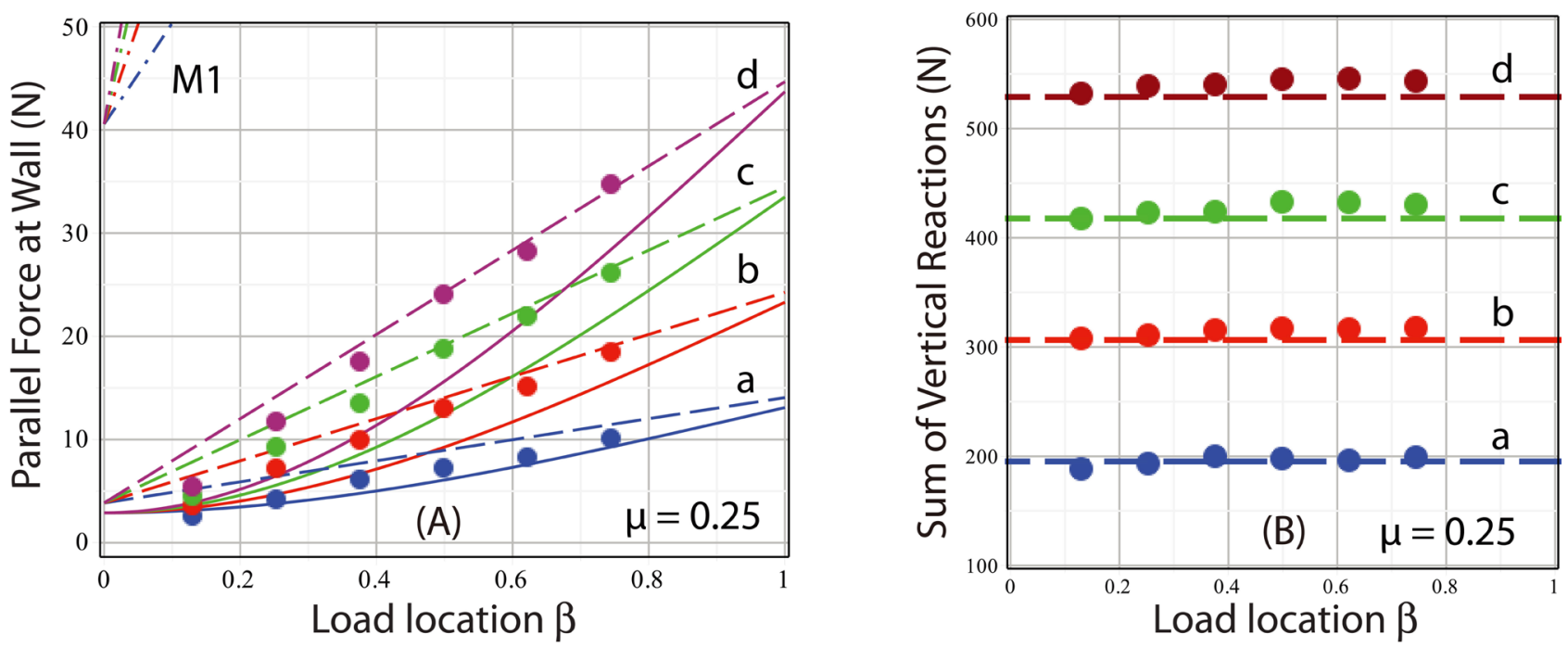

Figure 14. (A) Parallel reaction force at the wall $R_{4}$ (solid circles) deduced from the measurement of reaction $R_{3}$ normal to the wall and the experimental estimate of the coefficient of friction $\mu$ between the ladder and the wall. Line styles and colors of plots represent the same models and applied loads of Figure 11. M1 explicitly designates Model 1; (B) Test of the consistency of $R_{4}$ predicted by Model 3 (dashed line) for the applied loads $P$ of Figure 11 . For each load $P(25,50,75,100$ lbs), the reactions $R_{1}+\mu R_{3}$ should sum to a constant $W+P$, where $W$ is the ladder weight. 
- The elimination of Model 2 (and Model 1) likewise indicates that the bending of the ladder does not engender a torque at the ground support. Recall that it was this initially unknown moment that necessitated a complementary relation to resolve the static indeterminateness in the transverse direction. The reported experiment is consistent with no internal rotational moment at the base. This means that the ladder, initially presumed to be straight, has relaxed (very slightly) to the shape of an elastic deformation curve like those shown in Figure 10 in the course of reaching static equilibrium.

- The satisfactory accord between Model 3 and the experimental results tends to confirm that, for a ladder stationary, but not clamped, at its base, it is the friction at the wall that provides the complementary relation needed to resolve static indeterminateness so that all four forces of reaction can be calculated. The theoretical expressions for the reactions do not depend on the elastic modulus of the ladder. They do depend, however, on the coefficient of friction of the ladder at the wall. The experimental results are consistent with an empirical relation whereby the friction at a surface is proportional to the normal force on that surface.

The theoretical models tested in this paper each embodied one or more distinct physical attributes conceivably responsible for the forces of reaction on a fixed ladder modeled by a single beam. In this regard, the models were never intended to provide an exact theory of the stresses and strains within an actual ladder. To achieve that would require a detailed mechanical model, outside the scope of this paper, taking account of the exact shape, weight distribution, and area moments of inertia of the components of the ladder, including rungs. This caveat must be borne in mind when comparing theory and experiment in Figures 11-14. The dashed lines signifying Model 3 in the figures do not pass through all the experimental points, although they are sufficiently close for the purposes of distinguishing among the models. Small adjustments in the value of the coefficient of friction $\mu$ could achieve better agreement in one reaction or another, but there is little reason to make such refinements. Nor is there any reason to attempt to obtain an optimum value of $\mu$ by a statistical fitting process such as the method of maximum likelihood, or least square's analysis, or by Bayes' theorem [18]. A nonlinear fit of that kind would be computationally intensive and, assuming it even converged, would yield values of $\mu$ not much better than the estimate obtained simply and quickly by trial and inspection.

The great practical utility of Model 3, now that it is validated by experiment, is that it provides a simple, but satisfactory, way to estimate the forces exerted by ladders on structures as a function of the climber's weight and position of ascent and the ladder's angle of inclination. Moreover, it is readily generalizable in special situations of practical interest that may arise in engineering design or matters of litigation, such as cases where the ground is flat but sloped, or the wall is flat but not vertical, or the ladder inclines against a railing, not a wall, and overhangs it. 


\section{Effects of Impact Loads}

Although the primary focus of this paper has been on the static equilibrium of a ladder against a wall, the manner in which ladders are used in practice makes it necessary to discuss, albeit briefly, the question of impact loads. The experimental data of Table 1 were obtained by carefully and sequentially placing calibrated masses at designated rungs along the ladder so as to avoid imparting momentum to the ladder. Figure 15 shows the normal and parallel reactions (in arbitrary units) at the wall recorded as a function of time as a climber slowly ascends and then slowly descends the ladder, resting for a few seconds at each rung. The horizontal red bars mark the approximate values of the equilibrium reactions when the climber is stationary at each rung.

One sees, however, significant fluctuations about the equilibrium values as the climber transitions from one rung to the next. In particular, on the ascent, the reactions at the wall drop suddenly as the climber lifts his foot from a rung, and then rises suddenly as he places his foot on the next higher rung. On the descent, the reverse occurs for the normal reaction at the wall: the reaction increases dramatically as the climber raises his foot to step on the rung below.

The conveyance of momentum to a structure over a time interval is referred to as impact or impulse loading [19]. According to Newton's laws of motion, when the climber accelerates upward, the ladder must recoil downward, thereby exerting an additional force on whatever the ladder is leaning against. In physics, the integral of a force $F(t)$ over time $t$ is designated impulse. An essential

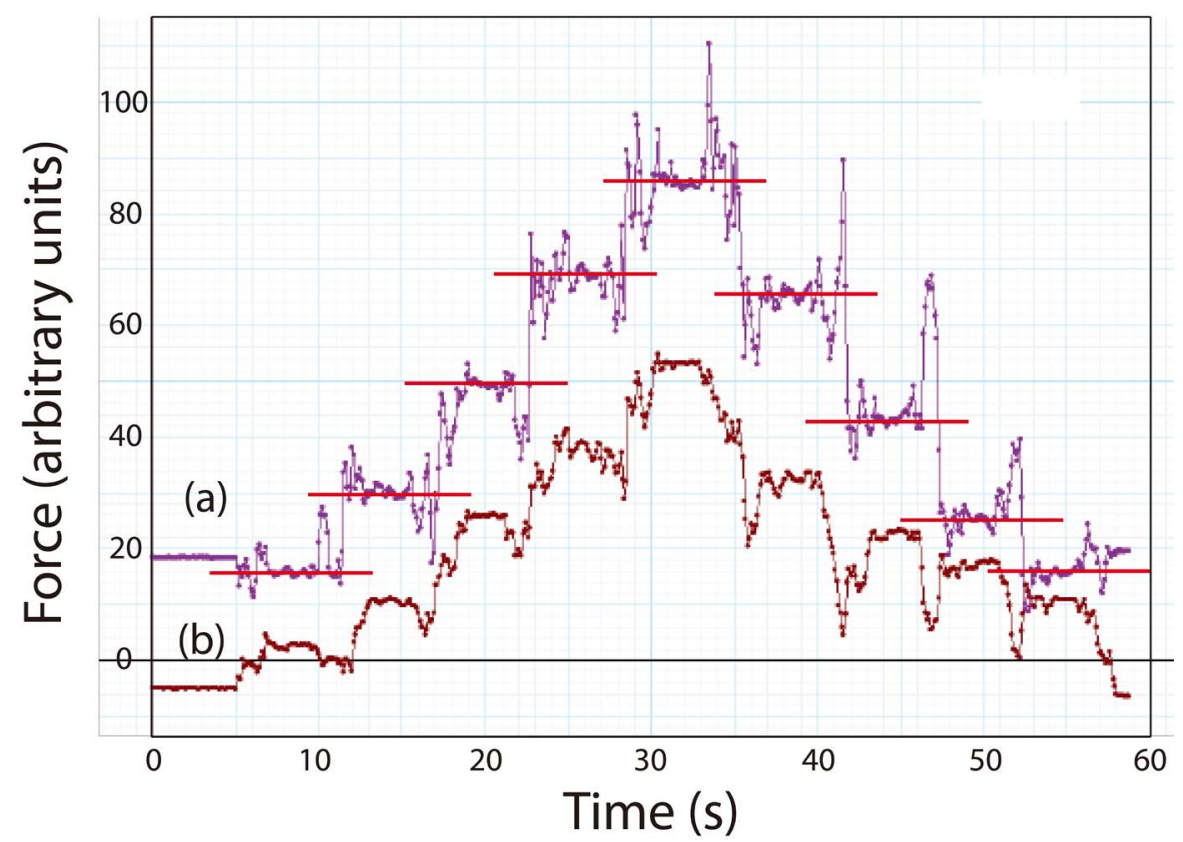

Figure 15. Normal (a) and parallel (b) forces at the wall as a function of time as a climber ascends, then descends, the first 5 rungs of a ladder. At each rung, the climber waits a few seconds until the condition of static equilibrium is achieved, as indicated by the horizontal red bars locating the mean equilibrium force (in arbitrary units) recorded at each rung. 
matter in the safe use of a ladder is to determine how large a force the impulsive loading by a climber creates in comparison to the forces of static equilibrium.

One way to do this is to model the ladder as linear elastic structure governed by Hooke's law

$$
P(t)=-k y(t)
$$

in which $k$ is the force constant and $y(t)$ is the deformation under the load $P(t)$. It is well known from the physics of oscillators [20] that the force constant $k$ and the oscillation angular frequency $\omega$ are related by

$$
k=M \omega^{2}
$$

where $M$ is the mass of the object. Newton's second law, in the absence of dissipation of mechanical energy, then takes the form

$$
\frac{\mathrm{d}^{2} y}{\mathrm{~d} t^{2}}+\omega^{2} y=\frac{P(t)}{M}
$$

for which the general solution, derivable by means of Green's functions [20], is

$$
y(t)=\frac{1}{M \omega} \int_{0}^{t} \sin \omega\left(t-t^{\prime}\right) P\left(t^{\prime}\right) \mathrm{d} t^{\prime}
$$

with initial conditions

$$
y(0)=\left.\frac{\mathrm{d} y}{\mathrm{~d} t}\right|_{t=0}=0 .
$$

The impulse load $P(t)$ can be modeled as a pulse of amplitude $P_{0}$ and width $\tau$ by the difference of two Heaviside functions

$$
P(t)=P_{0}[H(t)-H(t-\tau)] .
$$

Substitution of Equation (48) into (46) leads to the solution

$$
y(t)=\frac{P_{0}}{M \omega^{2}}[\cos \omega(t-\tau)-\cos \omega t]
$$

from which one obtains by standard calculus the maximum deflection

$$
y_{\text {max }}=\frac{P_{0}}{M \omega^{2}} \sqrt{2(1-\cos \omega \tau)}
$$

and therefore from Equations (43), (44) and (50) the maximum force of impact

$$
P_{\max }=\left|M \omega^{2} y_{\max }\right|_{\omega \tau=\pi}=2 P_{0} .
$$

$P_{0}$ is interpretable as the time-averaged load or, equivalently, the load at static equilibrium-i.e. the weight of the climber in the context of a ladder.

To the extent that the conditions of the pulse model realistically apply to a ladder, Equation (51) indicates that a climber can generate a maximum impact load that is twice his weight. Figure 16 shows a plot of the normal reaction at the ground (i.e. normal to a horizontal force platform) as a climber rapidly ascends and descends a ladder-in contrast to the slow ascent and descent of Figure 15 (note that the time base in Figure 16 is $1 / 3$ that of Figure 15). The blue horizontal bars mark the approximate reactions at static equilibrium at each rung. Of 


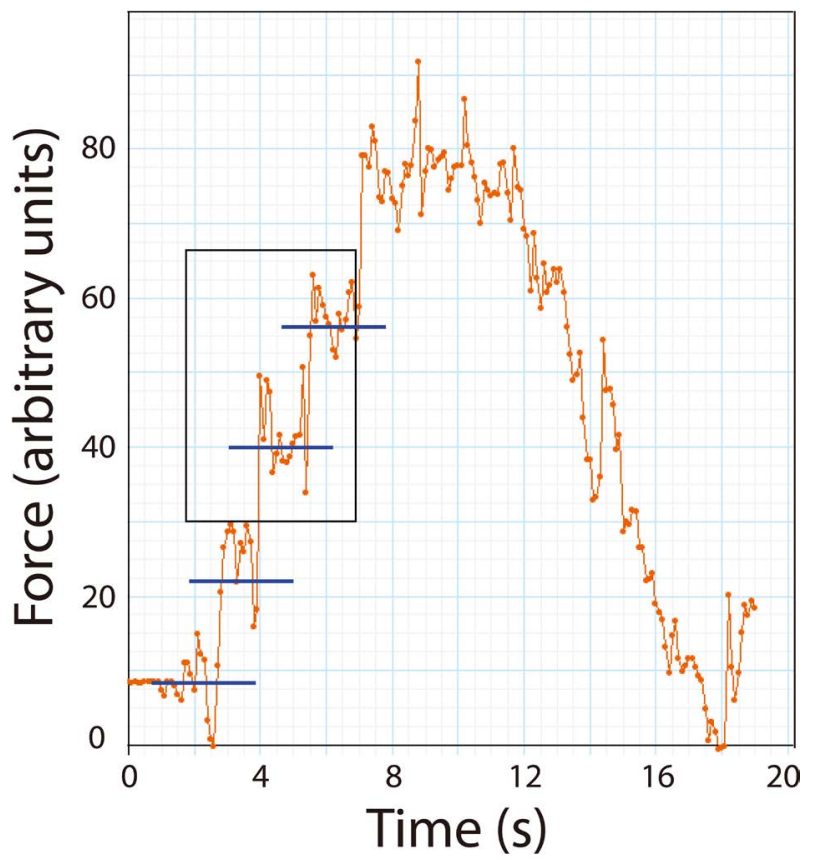

Figure 16. Normal force at the ground as a function of time as a climber ascends and descends rapidly. Horizontal blue bars locate the mean equilibrium force. Large fluctuations signify dynamic impact loads. The black rectangle encompasses the climber's transition from the third to fourth rung, thereby generating an impact load approximately one and half times his weight.

particular interest is the region within the black rectangle, which corresponds to the transition of the climber from rung 3 to rung 4 within a time interval $\Delta t$ of about $0.2 \mathrm{~s}$. Since the vertical separation between the rungs is $\Delta s=30 \mathrm{~cm}$, the vertical acceleration $a$ of the climber can be estimated from the kinematics of uniform acceleration [21] to be

$$
a=\frac{2 \Delta s}{(\Delta t)^{2}} \approx \frac{2 \times 0.3 \mathrm{~m}}{(0.2 \mathrm{~s})^{2}}=15 \mathrm{~m} \cdot \mathrm{s}^{-2}
$$

or, relative to the acceleration of gravity $g=9.81 \mathrm{~m} \cdot \mathrm{s}^{-2}$,

$$
\frac{a}{g}=\frac{P_{\text {climber }}}{P_{0}} \approx \frac{15}{10}=1.5 \text {. }
$$

Equation (53) is consistent with Equation (51) of the dynamical pulse model. Indeed, in a more comprehensive model, the dissipation of mechanical energy must be taken into account and would lead to a ratio $\left(P_{\max } / P_{0}\right)<2$. Nevertheless, the import of Equation (51) is that the numerical difference between impact forces and forces at static equilibrium can be consequential for those who use ladders in their work or who are responsible for setting safety guidelines and regulations with respect to ladders.

\section{Reaction Forces on a Single Wood Beam Inclined against a Wall}

The single-beam models developed in Section 2 to describe the reaction forces 
on a ladder inclined against a wall should, in principle, apply to an actual single beam inclined against a wall. The author is unaware of any previously reported experiment to measure these forces and determine which, if any, of the theoretical models best accounts for these forces. This section reports the results of such a test.

Measurements using one horizontal force platform at the base and one vertically mounted force platform at the top were made on a wood beam of mass $6.92 \mathrm{~kg}$, length $L=2.04 \mathrm{~m}$, width $w=7.5 \mathrm{~cm}$, height $h=8.5 \mathrm{~cm}$ (symbols correspond to panel $\mathrm{C}$ of Figure 1) inclined against a wall at an angle of $57.7^{\circ}$ to the ground. Starting at the center of the beam, round eyelets were screwed into the narrower side at intervals of $30 \mathrm{~cm}$ for a total of six eyelets. Loads were applied to the beam in units of $2 \mathrm{~kg}$ masses stacked on a $444 \mathrm{~g}$ weight hanger hooked to one of the eyelets. The eyelets, which served the equivalent function of rungs of the ladder, permitted load placements at fractional lengths of the beam

$$
\begin{aligned}
\beta & =\left(\frac{42}{204}, \frac{72}{204}, \frac{102}{204}, \frac{132}{204}, \frac{162}{204}, \frac{192}{204}\right) \\
& \approx(0.206,0.353,0.500,0.647,0.794)
\end{aligned}
$$

of which the first five eyelets were used in the experiment. Total loads (apart from the weight of the beam) ranged from about $4.4 \mathrm{~N}$ to $102.4 \mathrm{~N}$.

Table 2 records the measurements of the four forces of reaction. With a single beam, in contrast to a ladder, it was possible to measure directly the parallel force at the wall because the beam contacted the center of the vertical force platform where the shear-sensitive strain gauge was located.

Figure 17 shows a comparison of the measured reactions with the predictions of the three theoretical models for four different loads. One sees again, as in the case of the ladder measurements, that Model 1 (dashdot line) does not fit any of the data. For a coefficient of friction at the wall $\mu=0.35$, Models 2 and 3 give close predictions for the normal force at the ground (blue circles and curves) and the parallel force at the wall (maroon circles and curves), although the curve due to Model 3 (dashed line) passes through the data points (solid circles) and the curve due to Model 2 (solid line) does not. However, the parallel force at the ground (red circles and curves) and the normal force at the wall (green circles and curves) suffice to eliminate Model 2. The linear curve due to Model 3 (dashed line) very closely matches the measured points, whereas the curve due to Model 2 (solid line) has a marked curvature and passes well below the red solid circles. Only Model 3 accounts satisfactorily for all the data for a single value of the coefficient of friction.

This experiment lends strong support to the conclusion that the reaction forces on a single beam are described by the same theoretical model that describes the reaction forces on a fixed ladder, and that, as long as the base of either does not slip, the complementary relation needed to resolve the static indeterminateness of the problem is the force of friction at the wall. 
Table 2. Experimental normal and parallel reactions on a single wood beam.

\begin{tabular}{|c|c|c|c|c|c|}
\hline$\theta=57.7^{\circ}$ & LOAD $(\mathrm{N})$ & 43.55 & 63.15 & 82.75 & 102.35 \\
\hline REACTIONS (N) & EYELET & & & & \\
\hline \multicolumn{6}{|l|}{ Ground Normal $R_{1}$} \\
\hline & 1 & 100.3 & 118.8 & 137.7 & 156.6 \\
\hline & 2 & 99.6 & 118.6 & 137.3 & 154.6 \\
\hline & 3 & 100.0 & 119.2 & 134.1 & 150.9 \\
\hline & 4 & 98.0 & 115.8 & 131.8 & 149.0 \\
\hline & 5 & 99.0 & 113.8 & 131.9 & 148.3 \\
\hline \multicolumn{6}{|l|}{ Ground Parallel $R_{2}$} \\
\hline & 1 & 21.8 & 23.1 & 15.9 & 29.5 \\
\hline & 2 & 25.8 & 31.0 & 35.3 & 38.5 \\
\hline & 3 & 31.9 & 36.8 & 46.9 & 49.4 \\
\hline & 4 & 34.6 & 40.4 & 45.6 & 53.1 \\
\hline & 5 & 36.2 & 44.3 & 53.6 & 62.6 \\
\hline \multicolumn{6}{|l|}{ Wall Normal $R_{3}$} \\
\hline & 1 & 22.7 & 25.0 & 28.2 & 31.1 \\
\hline & 2 & 27.5 & 32.8 & 38.0 & 42.1 \\
\hline & 3 & 33.3 & 40.1 & 45.7 & 51.5 \\
\hline & 4 & 36.7 & 45.8 & 53.5 & 62.2 \\
\hline & 5 & 42.4 & 44.3 & 63.0 & 73.0 \\
\hline \multicolumn{6}{|l|}{ Wall Parallel $R_{4}$} \\
\hline & 1 & 9.0 & 9.7 & 10.6 & 10.9 \\
\hline & 2 & 9.2 & 10.3 & 10.7 & 13.0 \\
\hline & 3 & 9.1 & 9.7 & 13.5 & 16.2 \\
\hline & 4 & 10.7 & 12.8 & 16.1 & 18.4 \\
\hline & 5 & 10.4 & 14.3 & 15.9 & 18.7 \\
\hline
\end{tabular}

\section{Conclusions}

The question of how to calculate the four forces of reaction on a ladder in static equilibrium against a wall has been an unresolved issue for many years. The core of the difficulty is that the problem is statically indeterminate and requires complementary relations beyond the equations of static equilibrium. Three fundamental types of models, differing in the assumptions with regard to supports at the ground and wall, were examined. The comprehensive theoretical analysis and experimental measurements of this paper showed unambiguously that the forces of reaction were best accounted for by the model employing a single complementary relation characterizing friction at the wall.

The force law for friction that resolved the static indeterminateness in the third model is a single-parameter empirical relation. This parameter, the coefficient 

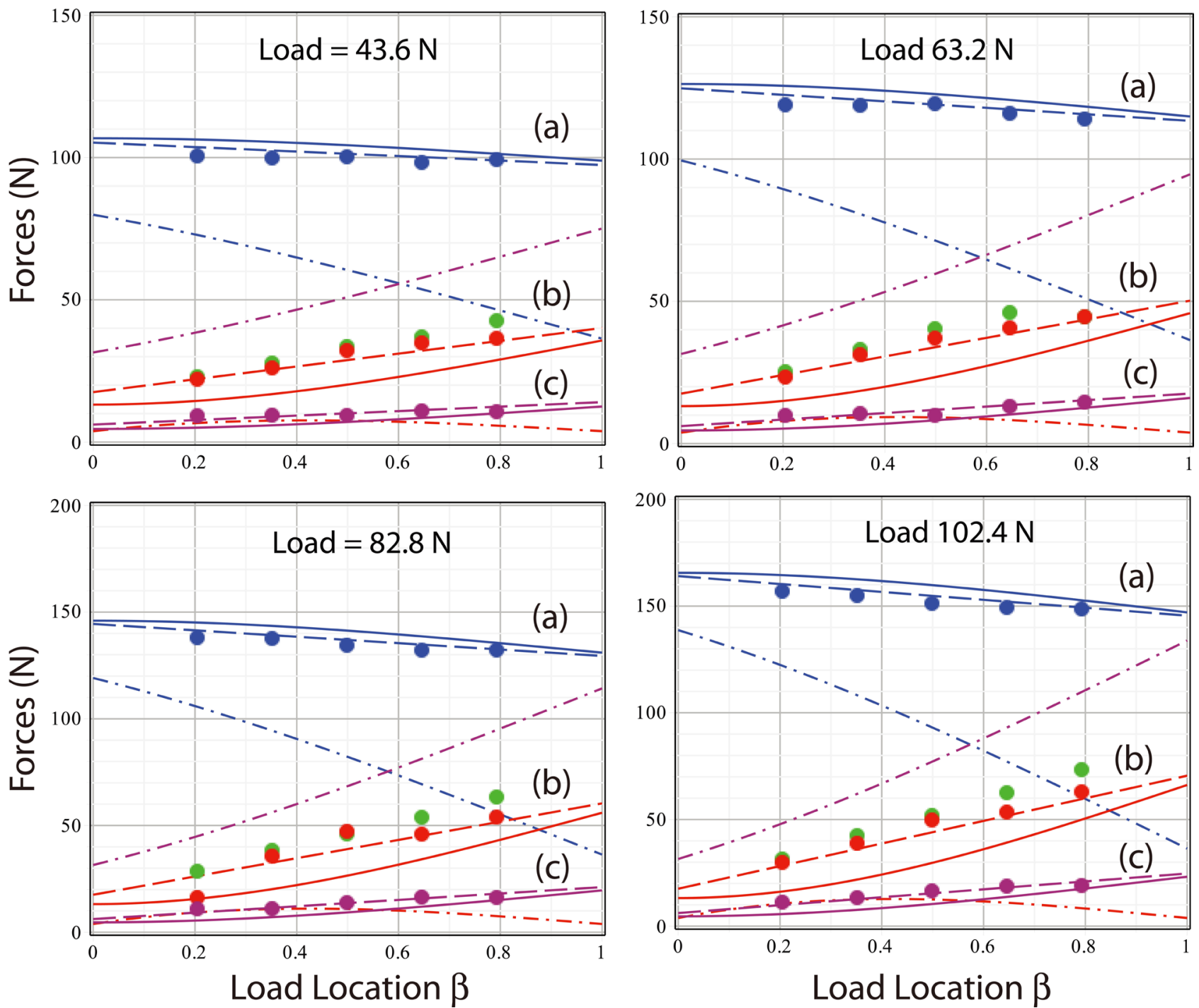

Figure 17. Reaction forces on a single wood beam for four different loads as a function of load location for an inclination angle of $57.7^{\circ}$ : (a) normal force at ground (blue), (b) parallel force at ground (red), (c) parallel force at wall (maroon). Measured points (solid circles) are superposed by predictions of Model 1 (dotdash), Model 2 (solid), Model 3 (dash). The green solid circles are experimental values of the normal force at the wall for which the theoretical curves are identical to those of (b). Parameters are beam weight $67.8 \mathrm{~N}$, beam length $204 \mathrm{~cm}$, and empirically found coefficient of friction 0.35 .

of friction $\mu$, was estimated from, and tightly constrained by, the experimental data. A critical test of the third model was, in fact, to show that a single value of this coefficient sufficed to yield the normal and parallel reactions at the ground and at the wall for all values and locations of the loads.

As part of the analyses reported here, the axial and transverse deformations of the single-beam ladder were calculated by means of Castigliano's theorem, and then confirmed by a full calculation of the deflection curve (elastica) of the beam. The analyses demonstrated that the deformation due to flexure is about two to three orders of magnitude larger than the deformation due to compression. Thus, any model that takes into account only axial compression, but neglects flexure, to resolve the static indeterminateness of reactions on an inclined beam is likely 
to be inaccurate.

Measurements were also made of the impact loading on a ladder by the ascent and descent of a climber. In a typical ascent without hesitation, the impact loading was found to be about $1 \frac{1}{2}$ times the climber's weight. This result is consistent with the predicted maximum value of 2 for the ratio of impact load to static load by a dynamic model of the ladder as a linear elastic medium subject to a pulse perturbation.

The successful description of the reaction forces on a ladder modeled as a single beam prompted the question of whether any of the three theoretical models satisfactorily accounted for the reaction forces on an actual single beam. Measurements analogous to those made on the ladder were also made on a wood beam of comparable length. The results showed that the same model that accounted for the reaction forces on the ladder also best predicted the reaction forces on the single wood beam. Whether this result is completely general, or whether a different outcome might arise for a beam of different composition, length, and/or cross section can only be determined by further experimentation.

A final point of importance concerns the inclusiveness of the three fundamental models. In other words, is there another independent way to analyze an Euler-Bernoulli (E-B) beam ladder that might produce better agreement with experiment than Model 3 of this paper? In the author's opinion, the answer is probably "no". The reason is that other models within the single E-B beam framework are either irrelevant, equivalent, or inconsistent.

For example, two models not analyzed in this paper include a ladder with simple (i.e. frictionless) support at the wall and a ladder with fixed support (i.e. infinite friction) at the wall. Neither model is relevant because the contact at the wall is neither frictionless nor immovable. (The models of this paper all assume non-slippage at the ground, for otherwise there would be no static equilibrium.)

Consider, however, a variation of Model 1. Model 1 resolved static indeterminateness by applying two boundary conditions to the wall support in the configuration of Panel B of Figure 1: no displacement axially (in the direction of force $A_{2}$ ) or transversely (in the direction of force $B_{2}$ ). Although this model was in marked disagreement with the experiment, a question might be raised as to whether the outcome could be different if the boundary conditions were applied to the wall support in the configuration of Panel A of Figure 1, which shows the usual orientation of a ladder. The modified boundary conditions would then be: no displacement vertically (in the direction of force $R_{4}$ ) or horizontally (in the direction of force $R_{3}$ ). To implement these boundary conditions, one would express the strain energy $U$ in terms of $R_{3}$ and $R_{4}$ (by transforming $A_{2}$ and $B_{2}$ ), then set to zero the derivatives of $U$ with respect to $R_{3}$ and $R_{4}$. This procedure, however, leads to exactly the same reactions as obtained in the original Model 1.

Another conceivable model might be to express $U$ in terms of $R_{3}$ and $R_{4}$ but impose only one boundary condition at the wall, e.g. no displacement verti- 
cally. The resulting equation, which couples the vertical and horizontal reactions at the wall, is then used together with Equation (31) which expresses zero torque in static equilibrium. This procedure is inconsistent, however, because the first equation leads to a rotational moment at the ground support, whereas the second equation describes the absence of such a moment. The resulting set of reactions depends on the length $(L)$ and width $(h)$ of the beam (in conflict with experimental results) and leads to large reaction forces that violate physical laws.

In conclusion, for any model of a ladder (or other physical structure) it is paramount that the equations of static equilibrium and the complementary relations be consistent and compatible with the boundary conditions.

\section{Acknowledgements}

E. Donovan, W. Emery, J. Hallquist, W. Scully, L. Sewanan, and W. Strange each helped take data at some point during the initial phase of this project. The author also thanks Trinity College for partial support through the research fund associated with the George A. Jarvis Chair of Physics.

\section{Conflicts of Interest}

The author declares no conflicts of interest regarding the publication of this paper.

\section{References}

[1] Ladders 101. https://www.americanladderinstitute.org/page/Ladders101

[2] Silverman, M.P. (2009) Final Report: Force of a Ladder on a Railing under Static and Dynamic Conditions. Case of Shattuck v. Wynfield, Hartford Superior Court, Hartford, CT.

[3] Knight, R.D. (2017) Physics for Scientists and Engineers: A Strategic Approach. 4th Edition, Pearson Education Inc., Boston, MA, 113.

[4] Mendelson, K.S. (1994) Statics of a Ladder Leaning against a Rough Wall. American Journal of Physics, 63, 148-150. https://doi.org/10.1119/1.17972

[5] Gonzalez, A.G. and Gratton, J. (1996) Reaction Forces on a Ladder Leaning against a Rough Wall. American Journal of Physics, 64, 1001-1005.

https://doi.org/10.1119/1.18317

[6] Hakkinen, K.K., Pesonen, J. and Rajamaki, E. (1988) Experiments on Safety in the Use of Portable Ladders. Journal of Occupational Accidents, 10, 1-19.

https://doi.org/10.1016/0376-6349(88)90002-8

[7] Lee, Y.H. and Tung, E.K. (1992) Body and Ladder Mechanical Stresses Analysis in a Climbing Strike. In: Kumar, S., Ed., Advances in Industrial Ergonomics and Safety $I V$, Taylor and French, Montreal, 1007-1014.

[8] Chang, W.-R., Chang, C.-C., Matz, S. and Son, D.H. (2004) Friction Requirements for Different Climbing Conditions in Straight Ladder Ascending. Safety Science, 42, 791-805. https://doi.org/10.1016/j.ssci.2004.02.002

[9] Silverman, M.P. and Farrah, J. (2018) Bending of a Tapered Rod: Modern Application and Experimental Test of Elastica Theory. World Journal of Mechanics, 8, 272-300. https://doi.org/10.4236/wjm.2018.87022 
[10] Hibbeler, R.C. (1997) Mechanics of Materials. 3rd Edition, Prentice Hall, Upper Saddle River, NJ, 582-583.

[11] Fowles, G.R. and Cassiday, G.L. (1993) Analytical Mechanics. 5th Edition, Harcourt Brace, New York, 54-55.

[12] Elastic Modulus of Aluminum. https://www.engineeringtoolbox.com/young-modulus-d_417.html

[13] Material Property Data. http://www.matweb.com/Search/MaterialGroupSearch.aspx?GroupID=316

[14] Elastic Modulus of Wood. https://www.engineersedge.com/lumber.htm

[15] Budynas, R.G. (1977) Advanced Strength and Applied Stress Analysis. McGraw-Hill, New York, 240-247.

[16] Matthews, J. and Walker, R.L. (1973) Mathematical Methods of Physics. W.A. Benjamin, Menlo Park, 107-109.

[17] Levien, R. (2008) The Elastica: A Mathematical History. Technical Report No. UCB/EECS 2008-103, Electrical Engineering and Computer Sciences University of California, Berkeley.

http://www.eecs.berkeley.edu/Pubs/TechRpts/2008/EECS-2008-103.html

[18] Silverman, M.P. (2014) A Certain Uncertainty: Nature's Random Ways. Cambridge University Press, Cambridge, 45-83. https://doi.org/10.1017/CBO9781139507370

[19] Paz, M. (1985) Structural Dynamics: Theory \& Computation. Van Nostrand Reinhold Co., New York, 63-65.

[20] Marion, J.B. and Thornton, S.T. (1988) Classical Dynamics of Particles and Systems. 3rd Edition, Harcourt Brace Jovanovich, New York, 98-135.

[21] Symon, K.R. (1971) Mechanics. 3rd Edition, Addison-Wesley, Reading, 11-13. 


\section{Appendix: Integration of the Heaviside Function}

The Heaviside function defined in Equation (12) and repeated below

$$
H\left(x-x_{0}\right)= \begin{cases}0 & x<x_{0} \\ 1 & x \geq x_{0}\end{cases}
$$

provides an economic way to represent piecewise continuous functions, such as frequently occur in the mechanics of continuous media. In the representation of static forces, impact forces, rotational moments, and strain energy densities it is often necessary to integrate over expressions of the form $z^{n} H(z-a)$ where $a$ is a non-negative constant and $n$ is a non-negative integer. The general result is

$$
\int_{0}^{x} z^{n} H(z-a) \mathrm{d} z=\left(\frac{x^{n+1}-a^{n+1}}{n+1}\right) H(x-a) \quad(n=0,1,2, \cdots) .
$$

A general proof, which will not be given here, can be constructed inductively from the examples below for $n=0,1$.

1) Case $n=0$ :

$$
\int_{0}^{x} H(z-a) \mathrm{d} z=\int_{0}^{x} H(z-a) \mathrm{d}(z-a)=\int_{-a}^{x-a} H(u) \mathrm{d} u=(x-a) H(x-a)
$$

Steps: Transform the integration variable from $z$ to $u=z-a$ and use the properties of $H(u)$ in Equation (54).

2) Case $n=1$ :

$$
\begin{aligned}
\int_{0}^{x} z H(z-a) \mathrm{d} z & =\int_{0}^{x}(z-a) H(z-a) d(z-a)+a \int_{0}^{x} H(z-a) d(z-a) \\
& =\int_{-a}^{x-a} u H(u) \mathrm{d} u+a \int_{-a}^{x-a} H(u) \mathrm{d} u \\
& =\frac{(x-a)^{2}}{2} H(x-a)+a[(x-a) H(x-a)] \\
& =\frac{x^{2}-a^{2}}{2} H(x-a)
\end{aligned}
$$

Steps:

- Line 1: Transform the integrand into a function of $u=z-a$ by appropriate subtraction and addition of terms.

- Line 2: Perform the first integral and import the result obtained in the case $n=0$ for the second integral.

- Lines 3 and 4: Expand and simplify the algebraic expressions to obtain the form of Equation (55). 